University of Nebraska - Lincoln

DigitalCommons@University of Nebraska - Lincoln

Nutrition \& Health Sciences Dissertations \& Theses

Nutrition and Health Sciences, Department of

Summer 7-26-2021

\title{
Bovine Mammary Alveolar MAC-T Cells Afford a Tool for Studies of Bovine Milk Exosomes in Drug Delivery.
}

Mojisola Ogunnaike

University of Nebraska-Lincoln, mogunnaike2@huskers.unl.edu

Follow this and additional works at: https://digitalcommons.unl.edu/nutritiondiss

Part of the Molecular, Genetic, and Biochemical Nutrition Commons

Ogunnaike, Mojisola, "Bovine Mammary Alveolar MAC-T Cells Afford a Tool for Studies of Bovine Milk Exosomes in Drug Delivery." (2021). Nutrition \& Health Sciences Dissertations \& Theses. 89.

https://digitalcommons.unl.edu/nutritiondiss/89

This Article is brought to you for free and open access by the Nutrition and Health Sciences, Department of at DigitalCommons@University of Nebraska - Lincoln. It has been accepted for inclusion in Nutrition \& Health Sciences Dissertations \& Theses by an authorized administrator of DigitalCommons@University of Nebraska Lincoln. 
Mojisola Ogunnaike

\author{
A THESIS \\ Presented to the Faculty of \\ The Graduate College at the University of Nebraska \\ In Partial Fulfillment of Requirements \\ For the Degree of Master of Science \\ Major: Nutrition and Health Sciences \\ Under the Supervision of Professor Janos Zempleni \\ Lincoln, Nebraska
}

July, 2021 


\title{
BOVINE MAMMARY ALVEOLAR MAC-T CELLS AFFORD A TOOL FOR STUDIES OF BOVINE MILK EXOSOMES IN DRUG DELIVERY.
}

\author{
Mojisola Ogunnaike, M.S. \\ University of Nebraska, 2021
}

Advisor: Janos Zempleni

Bovine milk exosomes (BMEs) have attracted attention as vehicles for delivering RNA therapeutics. BMEs originate in mammary alveolar cells. Here, we determined whether bovine mammary alveolar MAC-T cells are a promising tool to assess RNA delivery by BMEs. MAC-T cells exosomes (MAC-T BMEs) and BMEs were harvested by differential ultracentrifugation. Exosome size, morphology, microRNA content and marker proteins were assessed using nanoparticle tracking analysis, transmission electron microscopy, real-time PCR and immunoblot analysis, respectively. MAC-T cells were genetically engineered to secrete MAC-T BMEs endogenously labeled with a near-infrared fluorescent protein and tissue distribution was compared to fluorophore-labeled BMEs following intravenous injection in C57BL/6 mice. Morphology and size were similar in MAC-T BMEs and BMEs $(94 \pm 5.8 \mathrm{~nm}$ and $101 \pm 4.2 \mathrm{~nm}$, $p>0.05$ ). Both preparations expressed miR-320a, miR-200c and let-7a-5p (positive controls) but not miR-1 (negative control). Exosome marker proteins, CD9, CD63, CD81 and Tsg101, were detected in both MAC-T BMEs and BMEs. The distribution in mouse tissues was similar for both preparations, with liver being the primary accumulation site. Collectively, MAC-T BMEs are a promising tool for BMEs-based RNA delivery studies.

Key words: Bovine mammary alveolar MAC-T cells; bovine milk exosomes; CD81 fusion protein; drug delivery; RNA; transgenic 


\section{DEDICATION}

To God, the invisible creator of the universe. 


\section{ACKNOWLEDGEMENT}

I am deeply thankful to my Advisor, Dr. Janos Zempleni, for giving me the opportunity to be a part of his exciting research team at the University of Nebraska, Lincoln. Pursuing my master's program under his supervision provided me with the cutting-edge facilities and invaluable training that fostered my scientific growth - I am very grateful for this. I also appreciate my committee members, Dr. Mary Ann Johnson, and Dr. Audrey Atkin, for their vital suggestions and constructive guidance.

My sincere appreciation also goes to the past and present Zempleni lab members- Wei Zhao, Mengna Xia, John Wang, Sonal Sukreet, Afsana Khanam, Fang Zhou, Di Wu, Mahrou Sadri, Pearl Ebea, Alice Ngu, Shu Wang and Anthony Delaney for their resourcefulness and willingness to answer my questions. I am especially indebted to Dr. Wei Zhao and Dr. John Wang for their guidance and technical assistance.

My profound gratitude also goes to NHS administrative staff - Verona Skomski, Amy Brown, Diane Brown, Lori Rausch and the department chair, Dr. Mary Ann Johnson for their consistent support throughout my program.

Lastly, to the most important people in my life - my family and dear friends for their prayers and encouragements. I must specially thank Joshua Okafor for his timely help and invaluable lessons. 


\section{TABLE OF CONTENTS}

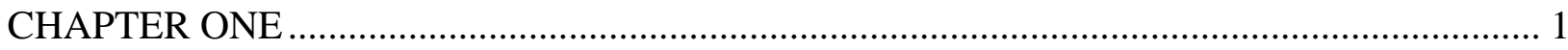

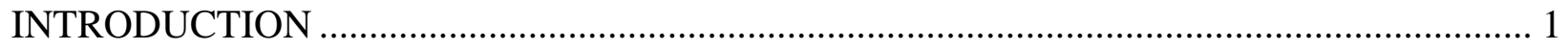

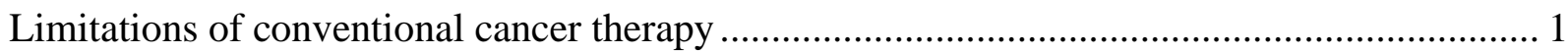

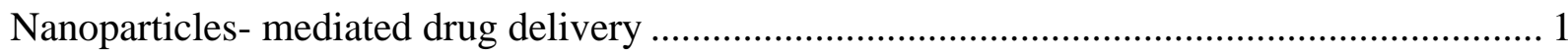

Bovine milk exosomes for drug delivery ............................................................................ 4

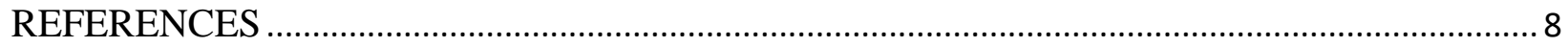

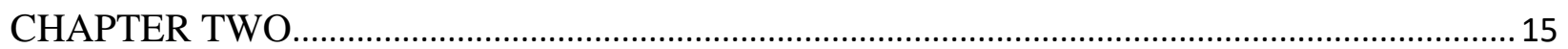

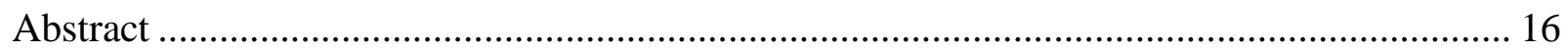

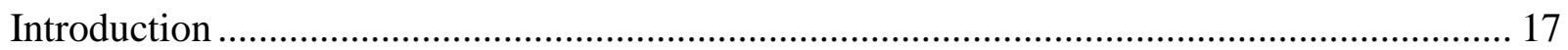

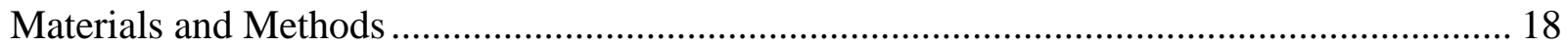

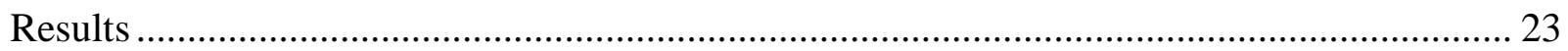

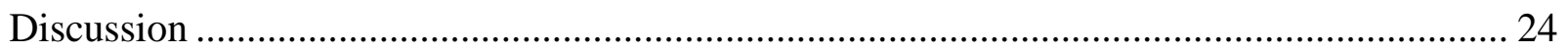

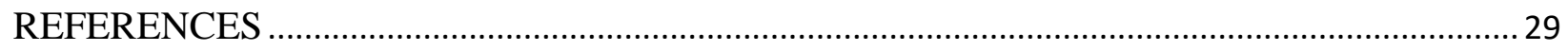

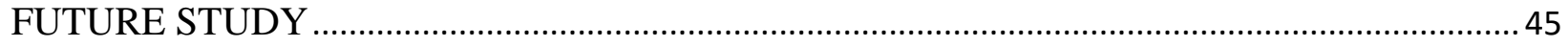

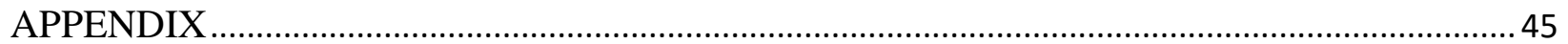




\section{LIST OF FIGURES}

\section{CHAPTER ONE}

Figure 1. 1 Formation of exosomes and their secretion into the extracellular............................ 3

Figure 1. 2 Exosomes sources and their advantages....................................................... 4

\section{CHAPTER TWO}

Figure 2. 1 Transmission electron microscopy images of MAC-T BMEs and BMEs 34

Figure 2. 2 Nanoparticle tracking analysis of MAC-T BMEs and BMEs ............................... 35

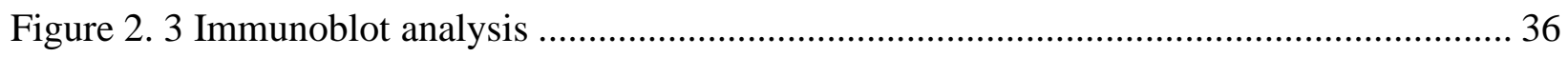

Figure 2. 4 Expression of a CD81-iRFP fusion protein..................................................... 37

Figure 2. 5 Authentication of engineered MAC-T BMEs ................................................. 38

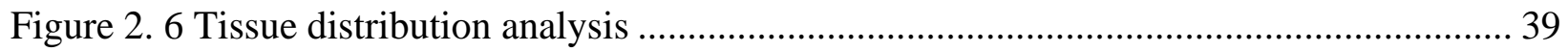

Figure S 1. Schematics illustration of CD81-iRFP vector construction .................................. 40

Figure S 2. Biodistribution of MAC-T BMEs and HiLyte BMEs in mice tissues. .................... 41 


\section{LIST OF TABLES}

TABLE 2. 1 MicroRNAs in MAC-T BMES and BMES.

TABLE S. 1 Primers used in real-time QPCR analysis ..................................................... 45

TABLE S. 2 Primers used in building the CD81-IRFP-HIS construct .................................... 44 


\section{CHAPTER ONE}

\section{INTRODUCTION}

\section{Limitations of conventional cancer therapy}

Although advanced understanding of cancer biology has led to the development of improved cancer therapeutics, cancer remains one of the leading causes of death worldwide due to unfavorable pharmacokinetics of therapeutics [1] [2].

Chemotherapy is the most common type of cancer therapy, and it employs free anticancer drugs and, more recently, RNA therapeutics (such as siRNA) to inhibit cancer progression [3]. siRNA is of particular importance because of their ability to stop tumor progression at the molecular level by knocking down key genes that govern cell proliferation, angiogenesis, metastasis and apoptosis [4]. However, the therapeutic index of free anticancer drugs and unmodified RNA therapeutics is suboptimal due to their non-specificity for tumor cells, rapid systemic clearance by the reticuloendothelial cells, unstable physicochemical properties, off target effects, rapid clearance by the reticuloendothelial cells, reduced therapeutic concentrations in target cells, and uniform distribution to both diseased and normal cells resulting in dose-limiting toxicity [5] [6] [7]. Hence, a protective and targeted delivery system is needed to improve their therapeutic effects [1].

\section{Nanoparticles- mediated drug delivery}

The application of nanoparticle mediated drug delivery significantly enhanced the therapeutic potential of therapeutics by increasing their systemic stability allowing for their increased accumulation at the target site [8]. Typically, nanoparticles are small sized $(20 \mathrm{~nm}-500 \mathrm{~nm})$ 
particles that possess interior core for drug packaging and an exterior core that can be decorated with functional peptides [9]. The small size of nanoparticles enables them to evade systemic clearance while their interior core confers stability and protection on encapsulated drugs and nucleic acids [10]. Synthetic lipid nanoparticles such as liposomes and polymeric micelles were the first to be explored in nanoparticle-based therapy and have been approved for clinical use [11]. Synthetic nanoparticles have substantially improved the efficacy of anticancer agents and therapeutics by increasing their therapeutic concentrations at the target site and reducing the offtarget side effects [6]. However, they are fraught with issues such as inefficient cargo delivery, formation of aggregates, induction of immune response, systemic toxicity, and poor stability [12]. In contrast, exosomes have natural ability to protect and deliver functional biomolecules to target cells with low immunogenicity [13]. Hence, they have recently gained traction in the pharmaceutical industry as potential drug packaging and delivery systems [14-16].

Exosomes are natural extracellular nanoparticles $(30-150 \mathrm{~nm})$ secreted by virtually all cell types for cell-to-cell communication. [17]. Their formation is highly coordinated: first, the endosomal membrane invaginates to form intraluminal vesicles (ILVs) enclosed within large multivesicular bodies (MVBs); these MVBs then either fuse with the plasma membrane to release ILVs into the extracellular space as exosomes or are mobilized for lysosomal degradation $[18,19]$. Before their release into the extracellular space, labile cargos such as miRNAs, lipids and proteins are selectively incorporated into exosomes [20] (Fig 1.). Intracellular communication is achieved through the interaction of exosomes with the surface of recipient cells or the delivery of regulatory cargos (miRNAs) to recipient cells [21, 22]. Exosomes have an advantage over synthetic 
nanoparticles in targeted drug delivery because they are more natural, stable, bioavailable and less toxic compared to synthetic nanoparticles. [23, 24].

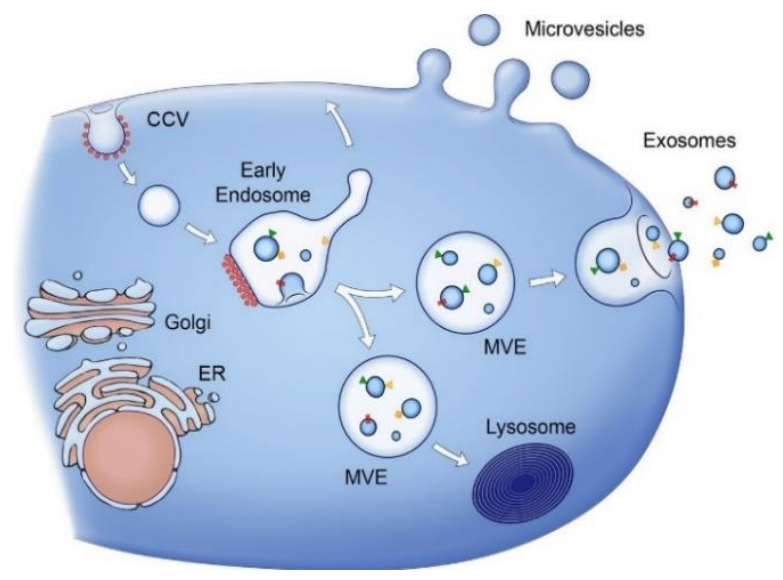

A

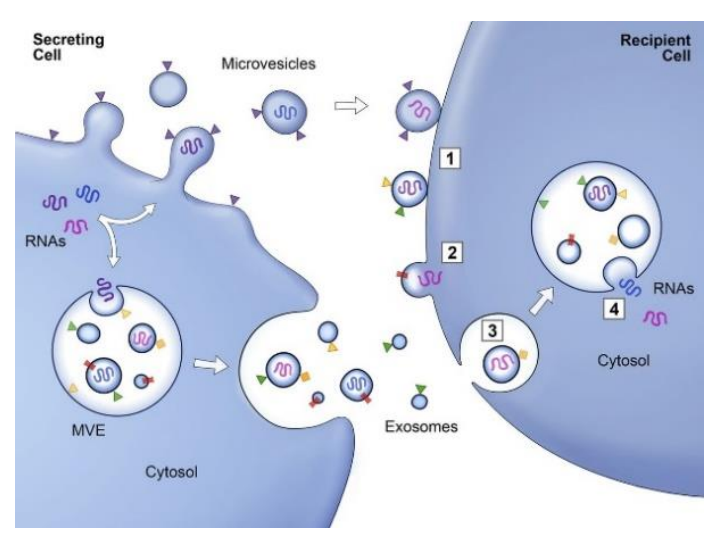

B

Figure 1. 1 Formation of exosomes and their secretion into the extracellular. Exosomes' uptake and cargo release in recipient cells space (A). Their release into the extracellular space (B). Adapted from Raposo et al., 2013 [25].

Exosomes from different sources such as milk, plasma and cell cultures have been explored for clinical translatability [24] (Fig. 2). This is necessary because exosomes possess a repertoire of cell-specific lipids and protein markers on their surface that determine their biological functions and tissue distribution pattern in-vivo [26]. For instance, tumor cell derived exosomes contain tumor associated biomolecules that induce metastatic behavior in normal cells [27]. Also, exosomes secreted by dendritic cells carry antigens that stimulate immune responses [28]. It is therefore crucial to carefully assess the biological properties and functions of exosomes and their progenitor cells so as to avoid occurrences that would jeopardize the intended therapeutic effects [26]. 
(1) Cancer cell derived exosomes

Target tumor cells

Induce metastatic behaviour

Immunogenic

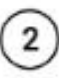

Low exosomes yied

Immunogenic
Dendritic cell derived exosomes

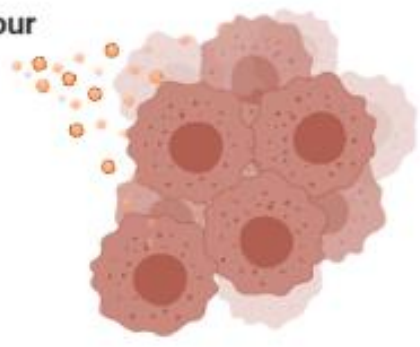

Dendritic cell derived exosomes
(3) Bovine milk exosomes

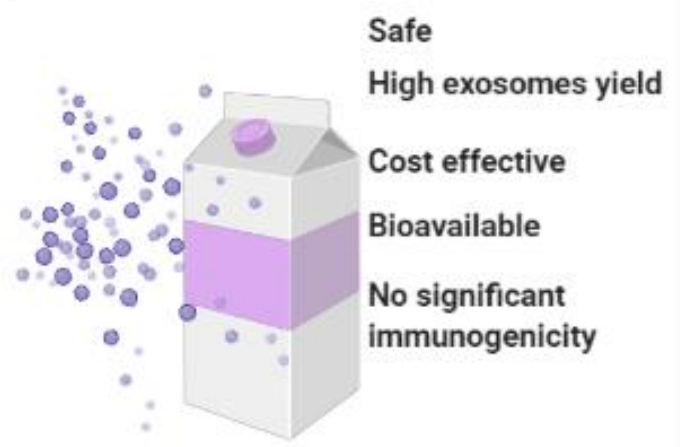

Figure 1. 2 Exosomes sources and their advantages. Bovine milk exosomes have superior drug delivery properties than exosomes secreted by cancer cells and dendritic cells. Illustration created with BioRender.com

\section{Bovine milk exosomes for drug delivery}

Bovine milk exosomes (BMEs) are extensively explored as promising vehicles for the delivery of RNA therapeutics due to their high yield, safety, cost effectiveness and natural ability to deliver functional biomolecules to target cells [29, 30]. BMEs are generally considered safe for drug delivery because of the prominent role of bovine milk in human nutrition [31]. Moreover, previous studies have reported that BMEs do not elicit significant immunogenic effect in humans and rats [32]. Bovine milk is cheap, readily accessible in most geographical regions and is a scalable source of exosomes [33]. Our lab has previously demonstrated that BMEs and their miRNA cargos are 
absorbed and transported by endocytosis in human endothelial cells [34]. Baier et al. were the first to report a postprandial increase in plasma concentration of milk miRNA following milk consumption [21]. Also, Manca et al. revealed that synthetic miRNAs encapsulated within BMEs could be delivered to the liver, intestinal tract, brain, and spleen in mice [35]. Mature miRNAs are non-coding RNAs ( 22 nucleotides in length) that can degrade mRNAs or inhibit their translation depending on their complementarity [36-38]. Approximately $60 \%$ of human genes are regulated by miRNAs [39]. BMEs can overcome placenta barrier [40]. Sadri et al., reported that BMEs-mediated delivery of synthetic miRNAs (miR-21-5p and miR-30d) elicited gene expression changes in murine placenta and increased fetal implantation [41]. It was recently reported that encapsulation of siRNA into BMEs facilitated their intracellular delivery and gene silencing activity in HEK293 cells [42]. These attributes make BMEs attractive vehicle for targeted delivery of drugs and therapeutic nucleic acids to diseased tissues.

The traditional approach of studying BMEs is fraught with huge variability and inefficient due to the challenging isolation and limited cargo loading procedure. The reason being that BMEs quality and their cargo profiles are significantly affected by several factors such as the physiological state of their parent cells, isolation techniques and sample processing [43]. The common isolation techniques such as ultracentrifugation, density gradient centrifugation, salting out and size exclusion allow enrichment of exosomes but with low purity as natural bovine milk contains stable multi-protein complexes that share comparable size with BMEs. Some free proteins even specifically complex with BMEs surface and co-precipitate during isolation procedure causing confounding effects on downstream analyses [44]. Also, the current loading of functional biomolecules into BMEs is passive and too inefficient for targeted drug delivery. Passive loading entails encapsulation of biomolecules into purified exosomes by electroporation, repeated freeze 
thawing, sonication, or even extrusion of natural cargos for a more functional one [45]. During this loading process, the exosomes membrane is temporarily disrupted and incubated with functional biomolecule which diffuses into the interior through the pores created on the exosomes. The major limitations of this method are as follows: (1) it is non-targeted; (2) loading efficiency depends on diffusion through a concentration gradient and the interaction between biomolecules and the phospholipid membrane of the exosomes; (3) It may lead to distortion of exosomes integrity. [26, 46, 47]

To accelerate BMEs studies for targeted therapeutic application, there is need to employ a more controlled in vitro cell system that will ensure reproducible isolation of pure BME preparations and allow functional biomolecules to be efficiently loaded into BMEs using gene technology [48]. Bovine mammary epithelial cell (MAC-T) is the functional unit of milk secretion and the primary origin of milk exosomes [49]. MAC-T cell is widely regarded as a reliable in vitro model for the studies of mammary gland functions because of its expression of milk-specific proteins [50] and thus is ideal for studying genetic modifications in BMEs.

Many studies have explored the surface modification of exosomes for imaging and targeting purposes using genetic engineering. Genetic engineering of donor cells for exosomal cargo loading offers the following benefits: ability to load encodable targeting ligands, imaging agents, antibodies or other functional biomolecule into exosomes with high efficiency, precision and specificity; minimal interference with exosomes surface proteins; and the flexible packaging of biomolecules in the interior and/or exterior surface of exosomes [48]. To genetically modify exosomes, the transgene is first fused to exosome-specific markers such as CD81, CD9 and CD63 etc. and then expressed in the donor cells by plasmid transfection. Transformed donor cells then secrete engineered exosomes with the functional protein attached to their surface [51]. For 
example, previous studies have produced brain targeting dendritic cell-derived exosomes by expressing a fusion of the central nervous system specific rabies viral glycoprotein (RVG) peptide, and exosomes specific protein, Lamp2b, in murine dendritic cells. The transformed dendritic cells secreted RVG-exosomes that crossed the blood-brain barrier (BBB). When loaded with $\beta$-site amyloid precursor protein cleaving enzyme 1 (BACE1) siRNAs, these engineered exosomes delivered their cargos to brain cells where they induced a $62 \%$ reduction in BACE1 mRNA expression [52]. Another study expressed a fusion protein consisting of ischemic myocardiumtargeting peptide (IMTP) and Lamp2b in bone marrow mesenchymal cells (BMSCs) and the transformed cells secreted engineered exosomes that showed increased uptake by ischemic myocardium compared to unmodified exosomes [53]. Aside from the packaging of targeting peptides, donor cells can be genetically modified to load highly sensitive fluorescent proteins into exosomes to visualize and monitor their biodistribution, viability, and biological activity/therapeutic efficiency in vivo [35, 54]. Traditionally, fluorescent lipophilic dyes are used to label BMEs, but this labelling approach is inefficient due to non-specificity, release of dyes from exosomes membranes, long half-life of chemical dyes and generation of non-exosomes related signals [55]. In contrast, gene labeling technology increases the stability and exosomespecificity of fluorescent proteins thereby providing an accurate assessment of the spatiotemporal distribution of exosomes [56, 57]. Some studies have endogenously labeled exosomes by fusing fluorescent transgenes such as eGFP, RFP, iRFP, Zsgreen1, exosomes-enriched markers such as CD63, CD9, CD81 [35, 58, 59]. 


\section{REFERENCES}

1. van der Meel, R., et al., Ligand-targeted particulate nanomedicines undergoing clinical evaluation: Current status. Advanced Drug Delivery Reviews, 2013. 65(10): p. 12841298.

2. Wicki, A., et al., Nanomedicine in cancer therapy: Challenges, opportunities, and clinical applications. Journal of Controlled Release, 2015. 200: p. 138-157.

3. Miele, E., et al., Nanoparticle-based delivery of small interfering RNA: challenges for cancer therapy. International journal of nanomedicine, 2012. 7: p. 3637-3657.

4. Strebhardt, K. and A. Ullrich, Paul Ehrlich's magic bullet concept: 100 years of progress. Nature Reviews Cancer, 2008. 8(6): p. 473-480.

5. Park, J.-H., et al., Cooperative nanomaterial system to sensitize, target, and treat tumors. Proceedings of the National Academy of Sciences of the United States of America, 2010. 107(3): p. 981-986.

6. Ulbrich, K., et al., Transferrin-and transferrin-receptor-antibody-modified nanoparticles enable drug delivery across the blood-brain barrier (BBB). European Journal of Pharmaceutics and Biopharmaceutics, 2009. 71(2): p. 251-256.

7. Housman, G., et al., Drug resistance in cancer: an overview. Cancers (Basel), 2014. 6(3): p. 1769-92.

8. Shaffer, C., Nanomedicine transforms drug delivery. Drug Discovery Today, 2005. 10(23): p. 1581-1582. 
9. Ahmad, A., et al., Precision Cancer Nanotherapy: Evolving Role of Multifunctional Nanoparticles for Cancer Active Targeting. Journal of Medicinal Chemistry, 2019. 62(23): p. 10475-10496.

10. Maeda, H., H. Nakamura, and J. Fang, The EPR effect for macromolecular drug delivery to solid tumors: Improvement of tumor uptake, lowering of systemic toxicity, and distinct tumor imaging in vivo. Adv Drug Deliv Rev, 2013. 65(1): p. 71-9.

11. Shi, X., K. Sun, and J.R. Baker, Spontaneous Formation of Functionalized DendrimerStabilized Gold Nanoparticles. The Journal of Physical Chemistry C, 2008. 112(22): p. $8251-8258$.

12. Jesorka, A. and O. Orwar, Liposomes: Technologies and Analytical Applications. Annual Review of Analytical Chemistry, 2008. 1(1): p. 801-832.

13. El-Andaloussi, S., et al., Exosome-mediated delivery of siRNA in vitro and in vivo. Nat Protoc, 2012. 7(12): p. 2112-26.

14. Kim, M.S., et al., Engineering macrophage-derived exosomes for targeted paclitaxel delivery to pulmonary metastases: in vitro and in vivo evaluations. Nanomedicine: Nanotechnology, Biology and Medicine, 2018. 14(1): p. 195-204.

15. Zempleni, J., Milk exosomes: beyond dietary microRNAs. Genes \& Nutrition, 2017. 12(1): p. 12.

16. Gurunathan, S., et al., Review of the Isolation, Characterization, Biological Function, and Multifarious Therapeutic Approaches of Exosomes. Cells, 2019. 8(4).

17. Gurunathan, S., et al., Review of the isolation, characterization, biological function, and multifarious therapeutic approaches of exosomes. Cells, 2019. 8(4): p. 307. 
18. Zhang, Y., et al., Exosomes: biogenesis, biologic function and clinical potential. Cell Biosci, 2019. 9: p. 19.

19. Patil, A.A. and W.J. Rhee, Exosomes: Biogenesis, Composition, Functions, and Their Role in Pre-metastatic Niche Formation. Biotechnology and Bioprocess Engineering, 2019. 24(5): p. 689-701.

20. Kalluri, R. and V.S. LeBleu, The biology, function, and biomedical applications of exosomes. Science, 2020. 367(6478).

21. Baier, S.R., et al., MicroRNAs are absorbed in biologically meaningful amounts from nutritionally relevant doses of cow milk and affect gene expression in peripheral blood mononuclear cells, HEK-293 kidney cell cultures, and mouse livers. J Nutr, 2014. 144(10): p. 1495-500.

22. Wolf, T., S.R. Baier, and J. Zempleni, The Intestinal Transport of Bovine Milk Exosomes Is Mediated by Endocytosis in Human Colon Carcinoma Caco-2 Cells and Rat Small Intestinal IEC-6 Cells. J Nutr, 2015. 145(10): p. 2201-6.

23. Munagala, R., et al., Bovine milk-derived exosomes for drug delivery. Cancer Lett, 2016. 371(1): p. 48-61.

24. Vader, P., et al., Extracellular vesicles for drug delivery. Advanced Drug Delivery Reviews, 2016. 106: p. 148-156.

25. Raposo, G. and W. Stoorvogel, Extracellular vesicles: exosomes, microvesicles, and friends. J. Cell Biol., 2013. 200(4): p. 373-383.

26. Luan, X., et al., Engineering exosomes as refined biological nanoplatforms for drug delivery. Acta Pharmacologica Sinica, 2017. 38(6): p. 754. 
27. Zomer, A., et al., In Vivo imaging reveals extracellular vesicle-mediated phenocopying of metastatic behavior. Cell, 2015. 161(5): p. 1046-1057.

28. Romagnoli, G.G., et al., Dendritic Cell-Derived Exosomes may be a Tool for Cancer Immunotherapy by Converting Tumor Cells into Immunogenic Targets. Frontiers in Immunology, 2015. 5(692).

29. Zempleni, J., Milk exosomes: beyond dietary microRNAs. Genes Nutr., 2017. 12: p. 12.

30. Munagala, R., et al., Bovine milk-derived exosomes for drug delivery. Cancer Lett., 2016. 371(1): p. 48-61.

31. Haug, A., A.T. Høstmark, and O.M. Harstad, Bovine milk in human nutrition--a review. Lipids Health Dis, 2007. 6: p. 25.

32. Mutai, E., A.E. Ramer-Tait, and J. Zempleni, MicroRNAs in bovine milk exosomes are bioavailable in humans but do not elicit a robust pro-inflammatory cytokine response. ExRNA, 2020. 2(1): p. 2.

33. Maghraby, M.K., et al., Extracellular vesicles isolated from milk can improve gut barrier dysfunction induced by malnutrition. Scientific Reports, 2021. 11(1): p. 7635.

34. Wolf, T., S.R. Baier, and J. Zempleni, The intestinal transport of bovine milk exosomes is mediated by endocytosis in human colon carcinoma caco-2 cells and rat small intestinal IEC-6 cells. J. Nutr., 2015. 145: p. 2201-2206.

35. Manca, S., et al., Milk exosomes are bioavailable and distinct microRNA cargos have unique tissue distribution patterns. Sci. Rep., 2018. 8(1): p. 11321.

36. Chen, K. and N. Rajewsky, The evolution of gene regulation by transcription factors and microRNAs. Nat. Rev. Genet., 2007. 8(2): p. 93-103. 
37. Jing, Q., et al., Involvement of microRNA in AU-rich element-mediated mRNA instability. Cell, 2005. 120(5): p. 623-634.

38. Djuranovic, S., A. Nahvi, and R. Green, miRNA-mediated gene silencing by translational repression followed by $m R N A$ deadenylation and decay. Science, 2012. 336(6078): p. 237-240.

39. Friedman, R.C., et al., Most mammalian mRNAs are conserved targets of microRNAs. Genome Res., 2009. 19(1): p. 92-105.

40. del Pozo-Acebo, L., et al., Bovine Milk-Derived Exosomes as a Drug Delivery Vehicle for miRNA-Based Therapy. International Journal of Molecular Sciences, 2021. 22(3): p. 1105.

41. Sadri, M., et al., Milk exosomes and miRNA cross the placenta and promote embryo survival in mice. Reproduction, 2020. 160(4): p. 501-509.

42. Warren, M.R., et al., Milk exosomes with enhanced mucus penetrability for oral delivery of siRNA. Biomaterials Science, 2021. 9(12): p. 4260-4277.

43. Ma, S., et al., Identification and characterization of differentially expressed exosomal microRNAs in bovine milk infected with Staphylococcus aureus. BMC Genomics, 2019. 20(1): p. 934.

44. Sedykh, S., A. Kuleshova, and G. Nevinsky, Milk Exosomes: Perspective Agents for Anticancer Drug Delivery. International Journal of Molecular Sciences, 2020. 21(18).

45. Akuma, P., O.D. Okagu, and C.C. Udenigwe, Naturally Occurring Exosome Vesicles as Potential Delivery Vehicle for Bioactive Compounds. Frontiers in Sustainable Food Systems, 2019. 3(23). 
46. Liu, C. and C. Su, Design strategies and application progress of therapeutic exosomes. Theranostics, 2019. 9(4): p. 1015-1028.

47. Johnsen, K.B., et al., A comprehensive overview of exosomes as drug delivery vehicles Endogenous nanocarriers for targeted cancer therapy. Biochim Biophys Acta, 2014. 1846(1): p. 75-87.

48. Adriano, B., et al., Milk exosomes: Nature's abundant nanoplatform for theranostic applications. Bioact. Mater., 2021. 6(8): p. 2479-2490.

49. Kandimalla, R., et al., Milk exosomes: A biogenic nanocarrier for small molecules and macromolecules to combat cancer. American Journal of Reproductive Immunology, 2021. 85(2): p. e13349.

50. Huynh, H.T., G. Robitaille, and J.D. Turner, Establishment of bovine mammary epithelial cells (MAC-T): an in vitro model for bovine lactation. Exp. Cell Res., 1991. 197(2): p. 191-199.

51. Liang, Y., et al., Engineering exosomes for targeted drug delivery. Theranostics, 2021. 11(7): p. 3183-3195.

52. Alvarez-Erviti, L., et al., Delivery of siRNA to the mouse brain by systemic injection of targeted exosomes. Nat Biotechnol, 2011. 29(4): p. 341-5.

53. Wang, X., et al., Engineered Exosomes With Ischemic Myocardium\&\#x2010;Targeting Peptide for Targeted Therapy in Myocardial Infarction. Journal of the American Heart Association, 2018. 7(15): p. e008737.

54. Sterzenbach, U., et al., Engineered exosomes as vehicles for biologically active proteins. Mol. Ther., 2017. 25: p. 1269-1278. 
55. Gangadaran, P., C.M. Hong, and B.-C. Ahn, Current Perspectives on In Vivo Noninvasive Tracking of Extracellular Vesicles with Molecular Imaging. BioMed Research International, 2017. 2017: p. 9158319.

56. Choi, H. and D.S. Lee, Illuminating the physiology of extracellular vesicles. Stem Cell Research \& Therapy, 2016. 7.

57. Yi, Y.W., et al., Advances in Analysis of Biodistribution of Exosomes by Molecular Imaging. International Journal of Molecular Sciences, 2020. 21(2).

58. Stickney, Z., et al., Development of exosome surface display technology in living human cells. Biochemical and Biophysical Research Communications, 2016. 472(1): p. 53-59.

59. Hyenne, V., O. Lefebvre, and J.G. Goetz, Going live with tumor exosomes and microvesicles. Cell Adhesion \& Migration, 2017. 11(2): p. 173-186. 


\section{CHAPTER TWO}

Bovine mammary alveolar MAC-T cells afford a tool for studies of bovine milk exosomes in drug delivery.

Mojisola Ogunnaike ${ }^{1}$ and Janos $\underline{\text { Zempleni }^{1}}$

${ }^{1}$ Department of Nutrition and Health Sciences, University of Nebraska-Lincoln, Lincoln, NE

Corresponding Author: Janos Zempleni; jzempleni2@unl.edu; 316C Leverton Hall, Lincoln, NE 68583, USA

Running Title: MAC-T cell exosomes as proxy for bovine milk exosomes

Word Count: 2864

Figures: 6

Table: 1

Supplementary Online Figures: 2

Supplementary Online Tables: 1

Declaration of Competing Interest:

J. Z. serves as consultant for PureTech Health, Inc. (Boston, MA). The authors declare that they have no known competing financial interest or personal relationships that could have appeared to influence the work reported in this paper.

Abbreviations: BMEs, bovine milk exosomes; iRFP, near-infrared fluorescent protein; MAC-T cells, mammary alveolar epithelial cells; MAC-T BMEs, MAC-T cell-derived bovine milk exosomes; ND, not detectable; RT qPCR, real time quantitative PCR; siRNA, small interfering RNA, 


\begin{abstract}
Bovine milk exosomes (BMEs) have attracted attention as vehicles for delivering RNA therapeutics. BMEs originate in mammary alveolar cells. Here, we determined whether bovine mammary alveolar MAC-T cells are a promising tool to assess RNA delivery by BMEs. MAC-T cells exosomes (MAC-T BMEs) and BMEs were harvested by differential ultracentrifugation. Exosome size, morphology, microRNA content and marker proteins were assessed using nanoparticle tracking analysis, transmission electron microscopy, real-time PCR and immunoblot analysis, respectively. MAC-T cells were genetically engineered to secrete MAC-T BMEs endogenously labeled with a near-infrared fluorescent protein and tissue distribution was compared to fluorophore-labeled BMEs following intravenous injection in C57BL/6 mice. Morphology and size were similar in MAC-T BMEs and BMEs $(94 \pm 5.8 \mathrm{~nm}$ and $101 \pm 4.2 \mathrm{~nm}$, $p>0.05$ ). Both preparations expressed miR-320a, miR-200c and let-7a-5p (positive controls) but not miR-1 (negative control). Exosome marker proteins, CD9, CD63, CD81 and Tsg101, were detected in both MAC-T BMEs and BMEs. The distribution in mouse tissues was similar for both preparations, with liver being the primary accumulation site. Collectively, MAC-T BMEs afford are a promising tool for BMEs-based RNA delivery studies.

Key words: Bovine mammary alveolar MAC-T cells; bovine milk exosomes; CD81 fusion protein; drug delivery; RNA; transgenic
\end{abstract}




\section{Introduction}

Nanoparticles are promising tools for delivering therapeutics, including the delivery of small interfering RNA (siRNA) to sites of disease such as tumors [1]. Nanotechnology offers a variety of advantages compared to the conventional method of delivering free drugs. For example, nanoparticles may facilitate the transfer of therapeutics across the blood-brain barrier and protect unstable RNA cargos against degradation [2, 3]. When self-derived exosomes from dendritic cells, modified by a brain homing peptide, rabies virus glycoprotein, were loaded with BACE1 siRNA and delivered by intravenous injection, the expression of BACE1 mRNA and protein decreased by $60 \%$ in neurons, microglia, oligodendrocytes and their precursors [4]. The technology was subsequently applied in the knockdown of other gene targets in mice and allelespecific knockdown of oncogenic KRAS ${ }^{\mathrm{G} 12 \mathrm{D}}$ in mouse models of pancreatic cancer [5-7]. While the production of cell culture-derived exosomes is a promising technology, their use in drug delivery poses challenges such as limited drug loading efficiency and lack of standardized isolation and purification methods [8].

Bovine milk exosomes (BMEs) have attracted attention for use in drug delivery because of the following observations. BMEs and their RNA cargos are bioavailable after oral administration and cross the blood-brain barrier (BBB) in humans, mice and pigs [9-11]. The cellular uptake of BMEs is facilitated by endocytosis in endothelial cells [12]. BMEs confer protection to RNA cargos against the harsh conditions encountered in the gastrointestinal tract (low pH, RNase) and in industrial processing of milk $[13,14]$. BMEs may be loaded with small RNAs by using calcium chloride and ethanol [11]. BMEs do not elicit a significant immune reaction in humans in vivo and ex vivo, and in rats $[15,16]$, which is an important consideration when pursuing federal approval of a drug [17]. The production of BMEs is scalable. On average, a cow 
produces $10,800 \mathrm{~kg}$ of milk annually in the U.S. in 2020, and milk contains approximately $10^{12}$ exosomes per milliliter $[13,18]$.

Here, we sought to establish a tool that accelerates the rate of discovery in drug delivery by BMEs by comparing morphology, size and content and tissue distribution in BMEs and exosomes secreted by bovine mammary alveolar MAC-T cells [19], and by assessing whether MAC-T cells and their exosomes are amenable to genetic engineering. MAC-T cells were chosen as cell model because evidence suggests that BMEs originate in bovine mammary alveolar cells $[20,21]$.

\section{Materials and Methods}

\section{Cell Cultures}

Bovine mammary alveolar MAC-T cells were derived from primary bovine mammary alveolar cells and immortalized using SV-40 large T antigen [19]. The MAC-T cells were a kind gift by Dr. Thomas McDonald at the University of Nebraska Medical Center. MAC-T cells were cultured as previously described [19]. Briefly, MAC-T cells were plated in T175 flasks (cat. \# 12-556-011, Thermo Scientific) at a density of $2.5 \times 10^{6}$ in Dulbecco's modified eagle medium (DMEM) (cat. \# SH30284.02, Cytiva) supplemented with 10\% fetal bovine serum (cat. \# S1150-

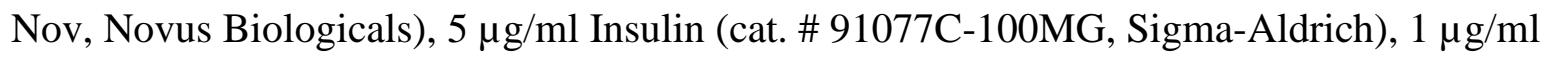
hydrocortisone (cat. \# H0396, Sigma-Aldrich) and 1\% penicillin-streptomycin mixture (cat. \# 17-602E, Lonza). The fetal bovine serum was conditioned by depleting exosomes through centrifugation at $120,000 \mathrm{~g}$ for 18 hours.

\section{Exosome Isolation and Authentication}

Exosomes were isolated from of MAC-T cell culture-conditioned media (containing exosomedepleted fetal bovine serum) and store bought bovine milk by differential ultracentrifugation 
using a F37L-8×100 rotor as previously described [10]. Hereafter, we refer to MAC-T cell derived exosomes as MAC-T BMEs. Protocol details are available through the EV-Track database (ID: EV210158). Purified exosome pellets were resuspended in $500 \mu \mathrm{l}$ of phosphate buffered saline and stored at $-80^{\circ} \mathrm{C}$ until analysis unless stated otherwise.

MAC-T BMEs and BMEs were thawed at room temperature and diluted as required for assessment of size and count by using a NanoSight NS300 tracking instrument (Malvern, Westborough, MA). We used the following instrument settings: camera level 14, detection threshold 5, syringe speed 100 and five 1-minute videos (technical replicates) per biological replicate $(\mathrm{n}=3)$. Data were analyzed by using NTA 2.3 software (Malvern). Morphology and dispersion of exosomes were assessed by using transmission electron microscopy [22]. Briefly, the exosomes were fixed, stained with phosphotungstic acid (3 mM), and analyzed as previously described using a Hitachi H7500 microscope in the Nebraska Microscopy Core at the University of Nebraska-Lincoln.

Exosomes were further authenticated by immunoblot analysis using positive and negative marker proteins as follows [23]. Protein extraction and analysis were performed as previously described by [10]. Protein concentrations were assayed by using the bicinchoninic acid assay (cat. \# HYK1001, MedChem Express) and $50 \mu \mathrm{g}$ total protein from exosomes and cell lysates were separated on 4-12\% Bis-Tris Protein Gels (cat. \# NP0322, Thermo fisher). Transblots on PVDF membranes were probed with the following antibodies: mouse anti-bovine CD9 (cat. \# GTX76185, Genetex), mouse anti-bovine CD63 (cat. \# MCA2042GA, BioRad), rabbit antihuman CD81 (cat. \# ab 155760, Santa Cruz) and rabbit anti-bovine TSG101 (cat. \# sc-7964, Santa Cruz) as exosome markers; and rabbit anti-mouse calnexin (cat. \# ab7580, Abcam,) as a negative control. Protein bands were visualized by using IRDye 800CW-conjugated goat anti- 
mouse (cat. \# 926-32210, LI-COR Biosciences) or IRDye 800CW-goat anti-rabbit (cat. \# 92632211, LI-COR Biosciences) and an Odyssey Clx Imaging System (LI-COR, Inc. Biosciences). MicroRNA Analysis

Quantitative real time PCR (qPCR) was used to assess the presence of microRNAs in MAC-T cell exosomes as previously described [24]. Cel-miR-39 was added to the samples during the isolation process as a spike-in control for the efficiency of RNA extraction and cDNA synthesis [9]. The expression levels of three milk enriched microRNAs, miR-320a, miR-200c and let-7a5p were assessed by qPCR using the universal reverse primer in the miScript SYBR Green PCR kit (cat. \# 218073, Qiagen) and microRNA-specific forward primers (Table S. 1). MiR-1 is not present in BMEs and was used as negative control [9]. Cycle threshold $(\mathrm{Ct})$ values greater than 29 were considered not detectable (N.D.) [25].

\section{Genetically Engineered MAC-T Cells}

MAC-T cells were genetically engineered to secrete transgenic exosomes containing a fusion protein composed of the tetraspanin CD81, near-infrared fluorescent protein (iRFP) and a polyhistidine tag (Supplementary Fig. 1). CD81 is a well-established exosome marker [26]. The feasibility of labeling CD81 through a fusion of a fluorescent protein to the C-terminus was previously demonstrated [27]. The design of the plasmid positions iRFP and polyhistidine inside exosomes, which minimizes the impact on the outer exosome surface and therefore tissue distribution [28]. iRFP has physicochemical properties conducive to successful tracking of exosomes in animals, e.g., minimal photodamage, low auto-fluorescence and deep tissue penetration $[29,30]$. To construct the plasmid, individual fragments of bovine CD81 (726 bp) and iRFP (978 bp) were amplified from MAC-T cell cDNA and piRFP plasmid (cat. \# 31857 , Addgene), respectively, using Q5 High-Fidelity DNA Polymerase kit (cat. \# M0491S, New 
England Biolabs) and the PCR primers shown in Supplementary Table 2. The open reading frame encoding the CD81-iRFP-polyhistidine fusion protein (1704 bp) was obtained by joining the PCR products using overlap PCR [31] with the primers denoted CD81_forward and iRFP_His reverse in Supplementary Table 2. The following program was used for overlap PCR: initial denaturation at $98^{\circ} \mathrm{C}$ for 2 minutes followed by 17 cycles of $98^{\circ} \mathrm{C}$ for 15 seconds (denaturing), $67^{\circ} \mathrm{C}\left(-0.5^{\circ} \mathrm{C} /\right.$ cycle) for 15 seconds (annealing) and $72^{\circ} \mathrm{C}$ for 30 seconds (extension). The first round of PCR was followed by another 23 cycles: $98^{\circ} \mathrm{C}$ for 15 seconds (denaturing), $59^{\circ} \mathrm{C}$ for 15 seconds (annealing), $72^{\circ} \mathrm{C}$ for 45 seconds (extension) and a final denaturation step at $72^{\circ} \mathrm{C}$ for 2 minutes. The product was cloned into a pCDH-puro lentiviral plasmid (cat. \# 46970, Addgene, Watertown, MA). Absence of mutations was confirmed by sequencing analysis and the plasmid is hereafter referred to as pCDH-CD81-iRFP (Supplementary Fig. 1).

The pCDH-CD81-iRFP plasmid, envelope plasmid (pMD2.G, cat. \# 12259, Addgene) and packaging plasmid (psPAX2, cat. \# 1226, Addgene) were co-infected into HEK-293T cells using attractene (cat. \# 301005, Qiagen), following the manufacturer's protocol, and lentiviral particles were collected by precipitation using standard PEG 8000 as previously described (Au - BaumlinSchmid et al. 2016). MAC-T cells were transfected with lentiviral particles using polybrene (cat \# 107689-10G, Sigma Aldrich) and stably transformed cells were selected using puromycin (2 $\mu \mathrm{g} / \mathrm{ml})$. MAC-T BMEs were collected by differential ultracentrifugation from 72-hour cultures of MAC-T cells and the expression of $i R F P$ in MAC-T BMEs and cells was assessed by using an Odyssey Clx Imaging system (LI-COR Biosciences, Inc.).

Tissue Distribution of MAC-T BMEs in Mice 
We compared the tissue distribution of iRFP-labeled MAC-T BMEs and HiLyte-labeled BMEs in C57BL/6 mice at 6 weeks of age (Jackson laboratory, stock number 000664) were assessed and compared. BMEs were labeled with carbonyl-reactive HiLyte 750 (cat. \# 61183, AnaSpec Inc., San Jose, CA) following the manufacturer's recommendations. We chose a dose of $1 \times 10^{11}$ exosomes/g body weight for tail vein injection of iRFP-labeled MAC-T cell BMEs, HiLyte 750labeled BMEs and controls (unlabeled MAC-T BMEs and unlabeled BMEs) based on the rationale that the dose produced a robust signal in a previous study [11]. The mice were euthanized 3 hours after injection when tissues were excised and washed with cold phosphate buffered saline. iRFP and HiLyte 750 fluorescence was analyzed using the 700-nm and 800-nm filters, respectively, in an Odyssey Clx Imaging System. The fluorescence signal was corrected by the signal from unlabeled controls. Densitometry analyses were performed by using LI-COR Image studio 5.2 software. The percent tissue distribution was calculated by dividing the fluorescence signal in individual tissues by the fluorescence signal from all tissues combined and multiplying that value by 100 . All animal procedures were conducted in accordance with the University of Nebraska-Lincoln's Institutional Animal Care and Use Committee and approved by the Institutional Animal Care Program (protocol 1713).

\section{Statistical Analyses}

Homogeneity of variances was assessed using Levene's test. Normality of distribution was confirmed by using the Shapiro-Wilk test. Data analysis was performed using unpaired student's t test. All statistical analyses were performed using Prism 6 (Graph pad software Inc.). Data are expressed as means \pm SEM as denoted in tables and figures. Differences were considered statistically significant if $P<0.05$. 


\section{Results}

\section{Characterization of MAC-T BMEs}

The morphology and size were similar in MAC-T BMEs and BMEs from milk. Both preparations presented as spherical membranous structures in TEM (Fig. 1). Nanoparticle tracking analysis revealed a homogenous particle distribution with a similar mean size of $94 \pm$ $5.8 \mathrm{~nm}$ and $101 \pm 4.2 \mathrm{~nm}$ for MAC-T exosomes and BMEs, respectively (Fig. 2). Both preparations expressed the exosome marker proteins CD9, CD63, CD81 and Tsg101 at the expected sizes, whereas a marker of microvesicles, calnexin, was detectable only in MAC-T cell lysate (Fig. 3).

\section{MicroRNA Content}

MAC-T BMEs and BMEs from milk expressed the three microRNAs we analyzed (positive controls, whereas miR-1 (negative control) was not detectable $(\mathrm{Ct}>29$ cycles) in any of the samples (Table 1). The expression of let-7a-5p and miR-200c was lower in MAC-T BMEs than in BMEs.

\section{Expression of CD81-iRFP-His in MAC-T cells and MAC-T BMEs}

MAC-T cells were transformed to express a CD81-iRFP-polyhistidine fusion protein, which localized to exosomes. Successful transformation and localization to exosomes was demonstrated as follows. Transformed MAC-T cell expressed iRFP, evidenced by the emission of near-infrared fluorescence (Fig. 4a). The image was taken after 8 passages of MAC-T cells, consistent with stable transformation. Media from cultures of transformed MAC-T cells produced strong near-infrared fluorescence suggesting secretion of the fusion protein by cells into the extracellular space (Fig. 4b); media from non-transformed MAC-T cells produced no fluorescence (control). iRFP fluorescence remained detectable in the supernatant from 16,000 $\mathrm{g}$ 
and $83,000 \mathrm{~g}$ centrifugation steps, but localized to the pellet following centrifugation at 130,000 $\mathrm{g}$ (Fig. 4c, depicting the pellet suspended in PBS). The vesicles in the 130,000 g pellet had a mean size of $82 \pm 1.2 \mathrm{~nm}$ (Fig. 5a) which is similar to exosomes secreted by non-transformed MAC-T cells (Fig. 2). The vesicles in the 130,000 g pellet tested positive for exosome markers, CD81 and Tsg101 (Fig. 5b). The position of the CD81 band was as expected for a CD81-iRFPpolyhistidine fusion protein, suggesting absence of degradation. The combination of centrifugation and size data, as well as protein markers suggests that the CD81-iRFPpolyhistidine fusion protein localized to exosomes.

\section{Tissue Distribution of Transformed MAC-T BMEs in C57BL/6 Mice}

The tissue distribution was similar for CD81-iRFP-positive MAC-T BMEs and HiLyte-labeled BMEs from milk following their intravenous injection in C57BL/6 mice. Both preparations accumulated primarily in the liver, but some accumulation was also apparent in the intestinal tract, lungs, kidneys, and spleen in both male and female mice (Fig. 6). The distribution of the two preparations differed in a few tissues. For example, the percent accumulation in brain and intestinal tract was higher for iRFP-labeled MAC-T BMEs than for HiLyte-labeled BMEs in both male and female mice. HiLyte-labeled BMEs produced a stronger fluorescence signal in murine tissues than iRFP MAC-T BMEs in both male and female mice (Supplementary Fig. 2).

\section{Discussion}

This is the first report introducing a bovine cell model amenable to genetic engineering for studies of BMEs in drug delivery. While the use of BMEs for delivering drugs has attracted considerable attention, previous modifications of BMEs were limited to the use of labeling and loading strategies $[11,16,32-34]$. MAC-T cells a promising tool for accelerating the rate of discovery in the field of BMEs and their use in nanotherapy. 
This study provides compelling evidence that morphology, size, and microRNA cargos are similar in MAC-T BMEs and BMEs, and exosomes from both sources have comparable tissue distribution profiles when administered intravenously to mice. MAC-T BMEs were authenticated by following recommendations by the International Society for Extracellular Vesicles (ISEV) [35]. Extensive authentication as well as the use of endogenous labeling approaches and the disclosure of protocol details in the EV-Track database enhance the rigor, reproducibility and transparency of this study.

$\mathrm{CD} 81$ is an exosome marker protein [26, 36]. Its choice for iRFP fusions, in addition to precipitation in the 130,000 $\mathrm{g}$ fraction during ultracentrifugation, absence of precipitation at low speed, morphology, size and marker proteins suggests that the CD81-iRFP localized to exosomes. The demonstration that MAC-T cells are amenable to genetic engineering makes them a potentially powerful tool in studies of nanotherapy.

This study adds to the existing body of evidence that milk exosomes originate in the mammary gland, which was previously supported by circumstantial evidence that epithelial cells are abundant in human milk and microRNA profiles are similar in BMEs in the lactating mammary gland in cows [20,21]. We acknowledge that this study does not provide an unambiguous answer to the question where BMEs originate, but that was not this study's goal. This study and the use of MAC-T cells has the following limitations. The amount of BMEs harvested from MAC-T cell cultures is small when done in a research laboratory setting. For example, we harvested approximately $10^{11}$ MAC-T BMEs from $350 \mathrm{~mL}$ of culture media in 3day cultures. That said, we achieved our primary goals of characterizing exosomes from transformed and non-transformed MAC-T cells and assessing the tissue distribution in mice, which informs future large-scale studies. Note that the accumulation of MAC-T BMEs in the 
murine brain was considerably less than the accumulation of (allogeneic) murine milk exosomes in the murine brain in our previous studies [11]. We propose that interactions between speciesspecific proteins on the exosome surface and the surface of receptor cells contributed to the observed difference. The role of protein-protein interactions in facilitating cellular exosome uptake has been reviewed [37]. Consistent with this theory, Wiklander et al. have enhanced the accumulation of exosomes in the brain through the expression of a brain homing signal, rabies virus glycoprotein [38]. Additional factors that might have contributed to the differences in brain accumulation include the route and timing of exosome administration, i.e., acute intravenous injection in this study compared to chronic oral intake in our previous study [11].

We conclude that MAC-T cells and BMEs are promising tools for optimizing drug delivery by milk exosomes. Future studies include the assessment of oral bioavailability and immunogenic potential of MAC-T BMEs.

\section{Credit authorship contribution statement}

Mojisola Ogunnaike: Formal analysis, Investigation, Writing - original draft. Janos Zempleni: Conceptualization, Study design, Supervision, Writing - review and editing, Funding acquisition.

\section{Acknowledgments}

The authors acknowledge the use of the Biomedical and Obesity Research Core (BORC) in the Nebraska Center for the Prevention of Obesity Disease through Dietary Molecules at the University of Nebraska-Lincoln. The BORC receives support from NIH (NIGMS) IDeA award 2P20GM104320. 
Funding: This work was supported by the National Institutes of Health [grant numbers 1P20GM104320]; the National Institute of Food and Agriculture [grant number 2016-6700125301, 2020-67017-30834]; and the United States Department of Agriculture [grant number Hatch-1011996, W4002] (to J.Z). 


\section{REFERENCES}

1. Mitchell, M.J., et al., Engineering precision nanoparticles for drug delivery. Nat. Rev. Drug Discov., 2021. 20(2): p. 101-124.

2. Ulbrich, K., et al., Transferrin- and transferrin-receptor-antibody-modified nanoparticles enable drug delivery across the blood-brain barrier (BBB). Eur. J. Pharm. Biopharm., 2009. 71(2): p. 251-256.

3. Zhang, C., et al., Inhibitory efficacy of hypoxia-inducible factor lalpha short hairpin RNA plasmid DNA-loaded poly (D, L-lactide-co-glycolide) nanoparticles on choroidal neovascularization in a laser-induced rat model. Gene Ther., 2010. 17(3): p. 338-351.

4. Alvarez-Erviti, L., et al., Delivery of siRNA to the mouse brain by systemic injection of targeted exosomes. Nat. Biotechnol., 2011. 29(4): p. 341-345.

5. Liu, Y., et al., Targeted exosome-mediated delivery of opioid receptor Mu siRNA for the treatment of morphine relapse. Sci. Rep., 2015. 5: p. 17543.

6. Zhou, Y., et al., Tumor-specific delivery of KRAS siRNA with iRGD-exoxomes efficiently inhibits tumor grwoth. BMC exRNA, 2019. 1: p. 28.

7. Kamerkar, S., et al., Exosomes facilitate therapeutic targeting of oncogenic KRAS in pancreatic cancer. Nature, 2017. 546(7659): p. 498-503.

8. Meng, W., et al., Prospects and challenges of extracellular vesicle-based drug delivery system: considering cell source. Drug Deliv., 2020. 27(1): p. 585-598.

9. Baier, S.R., et al., MicroRNAs are absorbed in biologically meaningful amounts from nutritionally relevant doses of cow's milk and affect gene expression in peripheral blood mononuclear cells, HEK-293 kidney cell cultures, and mouse livers. J. Nutr., 2014. 144: p. $1495-1500$. 
10. Wolf, T., S.R. Baier, and J. Zempleni, The intestinal transport of bovine milk exosomes is mediated by endocytosis in human colon carcinoma caco-2 cells and rat small intestinal IEC-6 cells. J. Nutr., 2015. 145: p. 2201-2206.

11. Manca, S., et al., Milk exosomes are bioavailable and distinct microRNA cargos have unique tissue distribution patterns. Sci. Rep., 2018. 8(1): p. 11321.

12. Kusuma Jati, R., et al., Human vascular endothelial cells transport foreign exosomes from cow's milk by endocytosis. Am. J. Physiol. Cell Physiol., 2016. 310: p. C800-C807.

13. Izumi, H., et al., Bovine milk contains microRNA and messenger RNA that are stable under degradative conditions. J. Dairy Sci., 2012. 95(9): p. 4831-4841.

14. Howard, K.M., et al., Loss of miRNAs during processing and storage of cow's (Bos taurus) milk. J. Agric. Food Chem., 2015. 63(2): p. 588-592.

15. Mutai, E., A.E. Ramer-Tait, and J. Zempleni, MicroRNAs in bovine milk exosomes are bioavailable in humans but do not elicit a robust pro-inflammatory cytokine response. BMC exRNA, 2020. 2: p. 2.

16. Munagala, R., et al., Bovine milk-derived exosomes for drug delivery. Cancer Lett., 2016. 371(1): p. 48-61.

17. United, S.D.o.H.a.H.S., et al., Nonclinical safety evaluation of the immunotoxic potential of drugs and biologics (draft guidance for induutry), Pharmacology/Toxicology, Editor. 2020: Rockville, MD. p. 1-10.

18. Statista, Milk produced per cow in the United States from 1999 to 2020 (in pounds). 2021. 
19. Huynh, H.T., G. Robitaille, and J.D. Turner, Establishment of bovine mammary epithelial cells (MAC-T): an in vitro model for bovine lactation. Exp. Cell Res., 1991. 197(2): p. 191-199.

20. Alsaweed, M., et al., Human milk miRNAs primarily originate from the mammary gland resulting in unique miRNA profiles of fractionated milk. Sci. Rep., 2016. 6: p. 20680.

21. Le Guillou, S., et al., Characterization of Holstein and Normande whole milk miRNomes highlights breed specificities. Sci. Rep., 2019. 9(1): p. 20345.

22. Sukreet, S., et al., Isolation of extracellular vesicles from byproducts of cheese making by tangential flow filtration yields heterogeneous fractions of nanoparticles. J. Dairy Sci., 2021. in press.

23. Lotvall, J., et al., Minimal experimental requirements for definition of extracellular vesicles and their functions: a position statement from the International Society for Extracellular Vesicles. J. Extracell. Vesicles, 2014. 3: p. 26913.

24. Leiferman, A., et al., Storage of extracellular vesicles in human milk, and microRNA profiles in human milk exosomes and infant formulas. J. Pediatr. Gastroenterol. Nutr., 2019. 69(2): p. 235-238.

25. Wang, L., et al., RNase H2-dependent polymerase chain reaction and elimination of confounders in sample collection, storage, and analysis strengthen evidence that microRNAs in bovine milk are bioavailable in humans. J. Nutr., 2018. 148(1): p. 153-159.

26. Kowal, J., et al., Proteomic comparison defines novel markers to characterize heterogeneous populations of extracellular vesicle subtypes. Proc. Natl. Acad. Sci. USA, 2016. 113(8): p. E968-977. 
27. Stickney, Z., et al., Development of exosome surface display technology in living human cells. Biochemical and Biophysical Research Communications, 2016. 472(1): p. 53-59.

28. Andreu, Z. and M. Yanez-Mo, Tetraspanins in extracellular vesicle formation and function. Front. Immunol., 2014. 5: p. 442.

29. Yuan, L., et al., A unique class of near-infrared functional fluorescent dyes with carboxylic-acid-modulated fluorescence ON/OFF switching: rational design, synthesis, optical properties, theoretical calculations, and applications for fluorescence imaging in living animals. J. Am. Chem. Soc., 2012. 134(2): p. 1200-1211.

30. Filonov, G.S., et al., Bright and stable near-infrared fluorescent protein for in vivo imaging. Nat. Biotechnol., 2011. 29(8): p. 757-761.

31. Nakamura, M., et al., Minimum GC-Rich Sequences for Overlap Extension PCR and Primer Annealing, in DNA Cloning and Assembly Methods, S. Valla and R. Lale, Editors. 2014, Humana Press: Totowa, NJ. p. 165-181.

32. Agrawal, A.K., et al., Milk-derived exosomes for oral delivery of paclitaxel. Nanomedicine, 2017. 13(5): p. 1627-1636.

33. Aqil, F., et al., Milk exosomes - Natural nanoparticles for siRNA delivery. Cancer Lett., 2019. 449: p. 186-195.

34. Grossen, P., et al., Evaluation of bovine milk extracellular vesicles for the delivery of locked nucleic acid antisense oligonucleotides. Eur. J. Pharm. Biopharm., 2021.

35. Thery, C., et al., Minimal information for studies of extracellular vesicles 2018 (MISEV2018): a position statement of the International Society for Extracellular Vesicles and update of the MISEV2014 guidelines. J. Extracell. Vesicles, 2018. 7(1): p. 1535750. 
36. Colombo, M., et al., Analysis of ESCRT functions in exosome biogenesis, composition and secretion highlights the heterogeneity of extracellular vesicles. J. Cell Sci., 2013. 126(Pt 24): p. 5553-5565.

37. Gonda, A., et al., Internalization of exosomes through receptor-mediated endocytosis. Mol. Cancer Res., 2019. 17(2): p. 337-347.

38. Wiklander, O.P., et al., Extracellular vesicle in vivo biodistribution is determined by cell source, route of administration and targeting. J. Extracell. Vesicles, 2015. 4: p. 26316. 
MAC-T BMEs

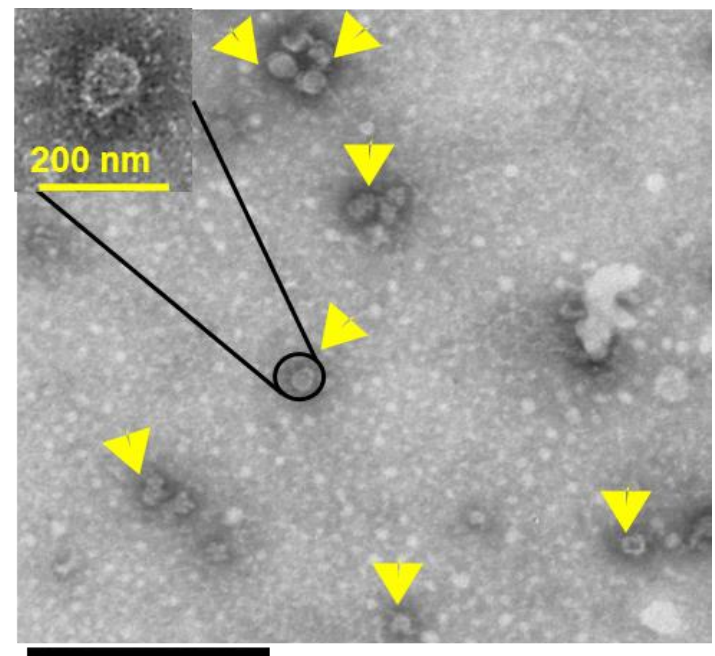

$800 \mathrm{~nm}$
BMES

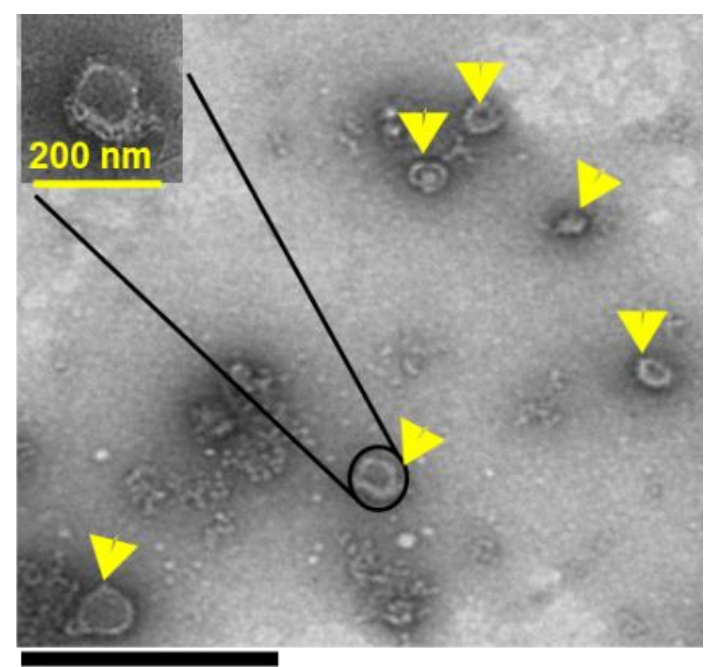

$800 \mathrm{~nm}$

Figure 2. 1 Transmission electron microscopy images of MAC-T BMEs and BMEs. Large field:

5,000-fold magnification; inserts: 20,000-fold magnification. 

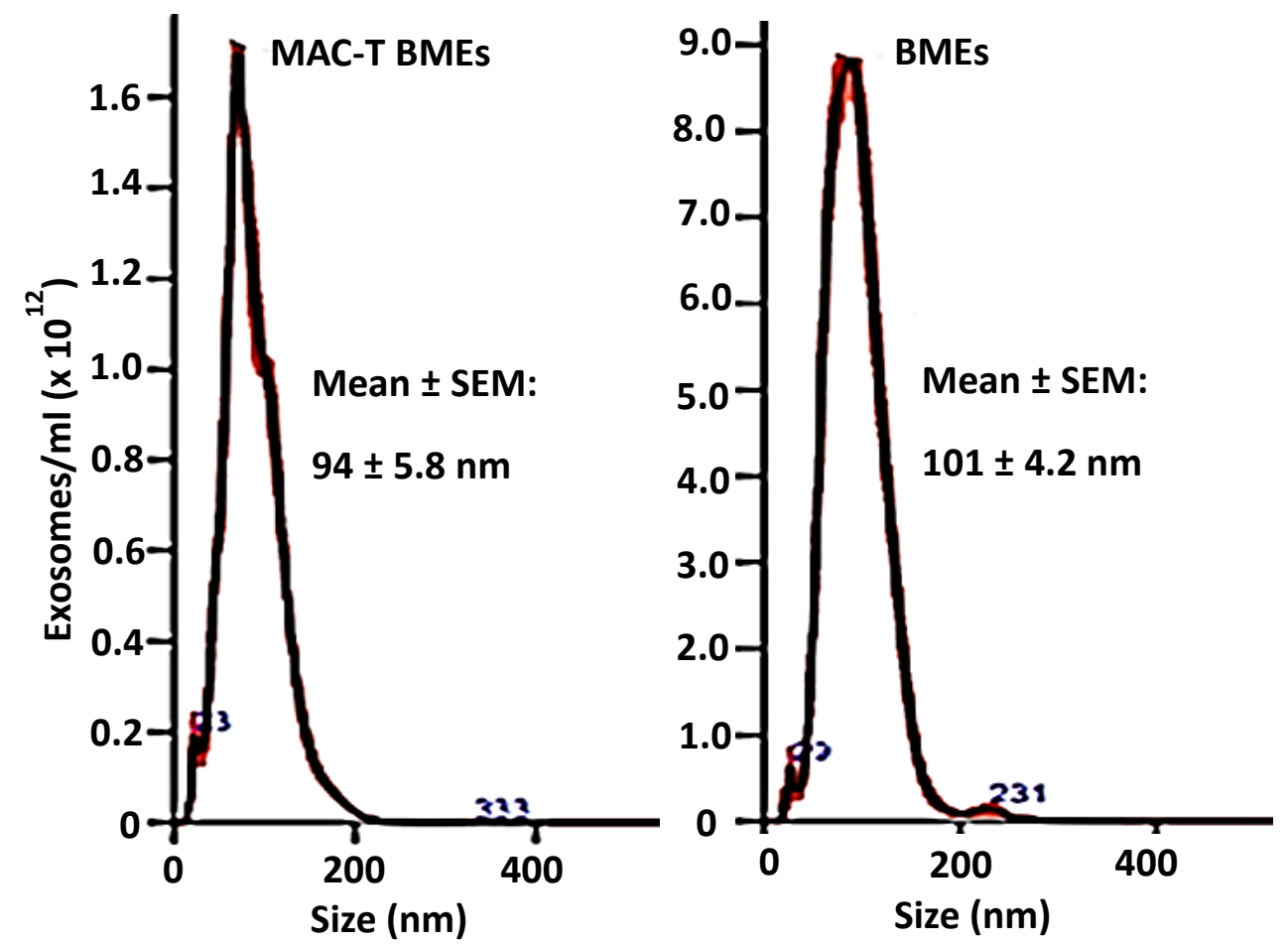

Figure 2. 2 Nanoparticle tracking analysis of MAC-T BMEs and BMEs. Values are expressed as mean \pm SEM. $\mathrm{p}>0.05(\mathrm{n}=3)$. 


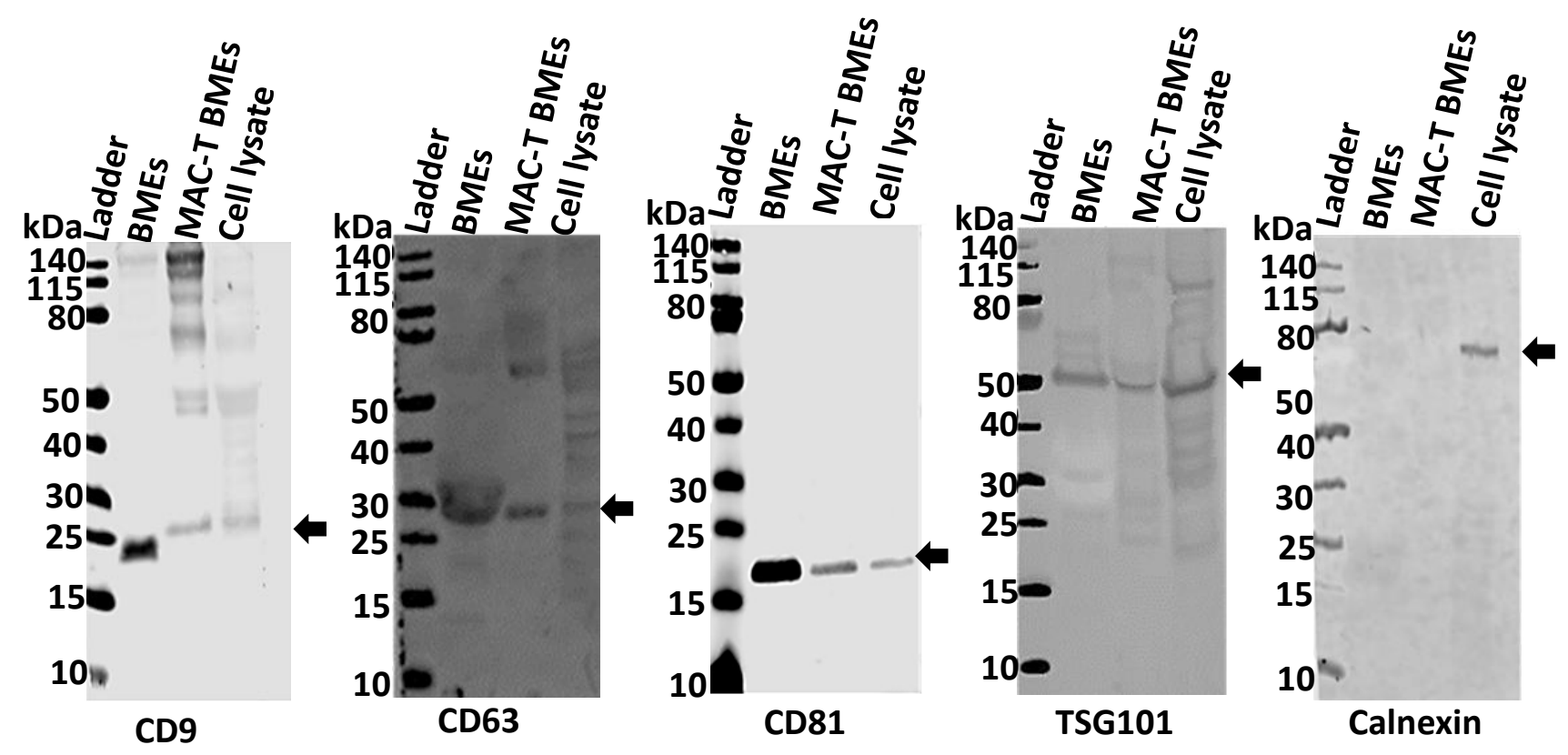

Figure 2. 3 Immunoblot analysis of protein extracts from BMEs, MAC-T BMEs, and MAC-T cell lysate. CD9, CD63, CD81 and Tsg101 are exosome markers; calnexin is microvesicle marker. 
(A)

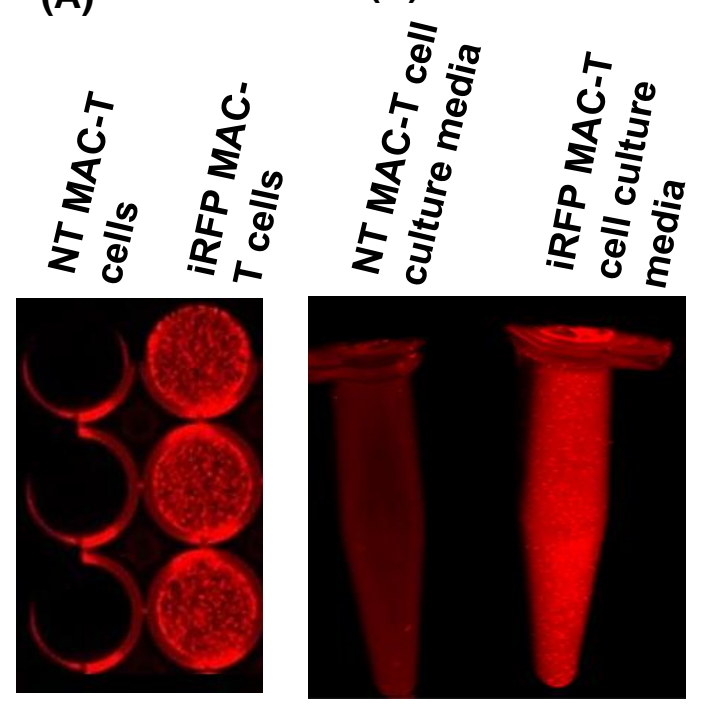

(C)

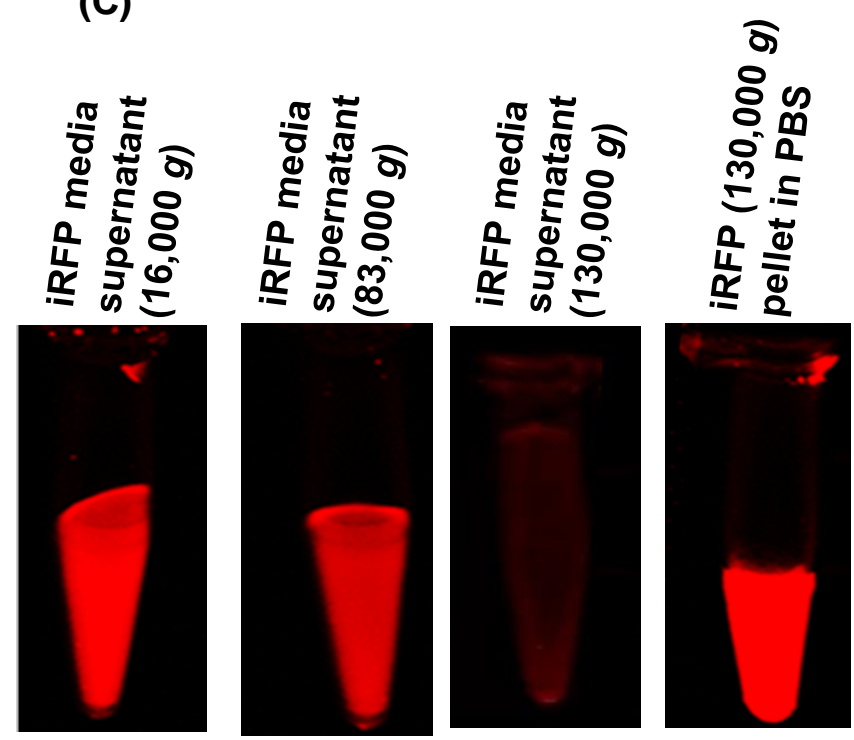

Figure 2. 4 Expression of a CD81-iRFP fusion protein in genetically engineered MAC-T cells and their exosomes. (a) Expression of CD81-iRFP in non-transformed (NT) and transformed MAC-T cells after 8 passages. (b) CD81-iRFP in media from non-transformed and transformed MAC-T cells. (c) Localization of CD81-iRFP signal to centrifugation fractions. 
(A)

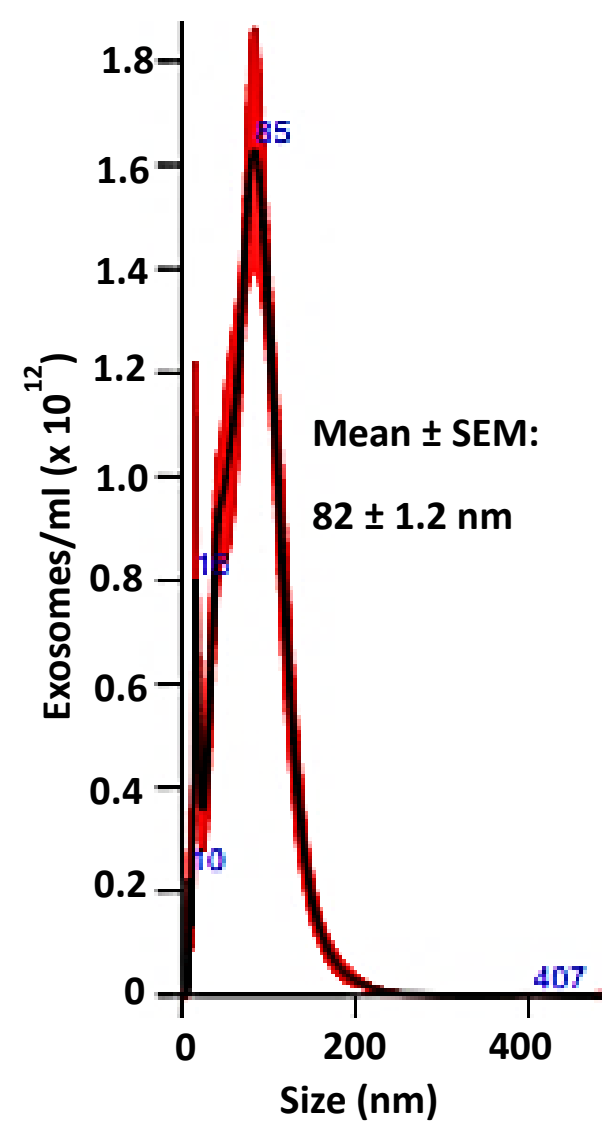

(B)

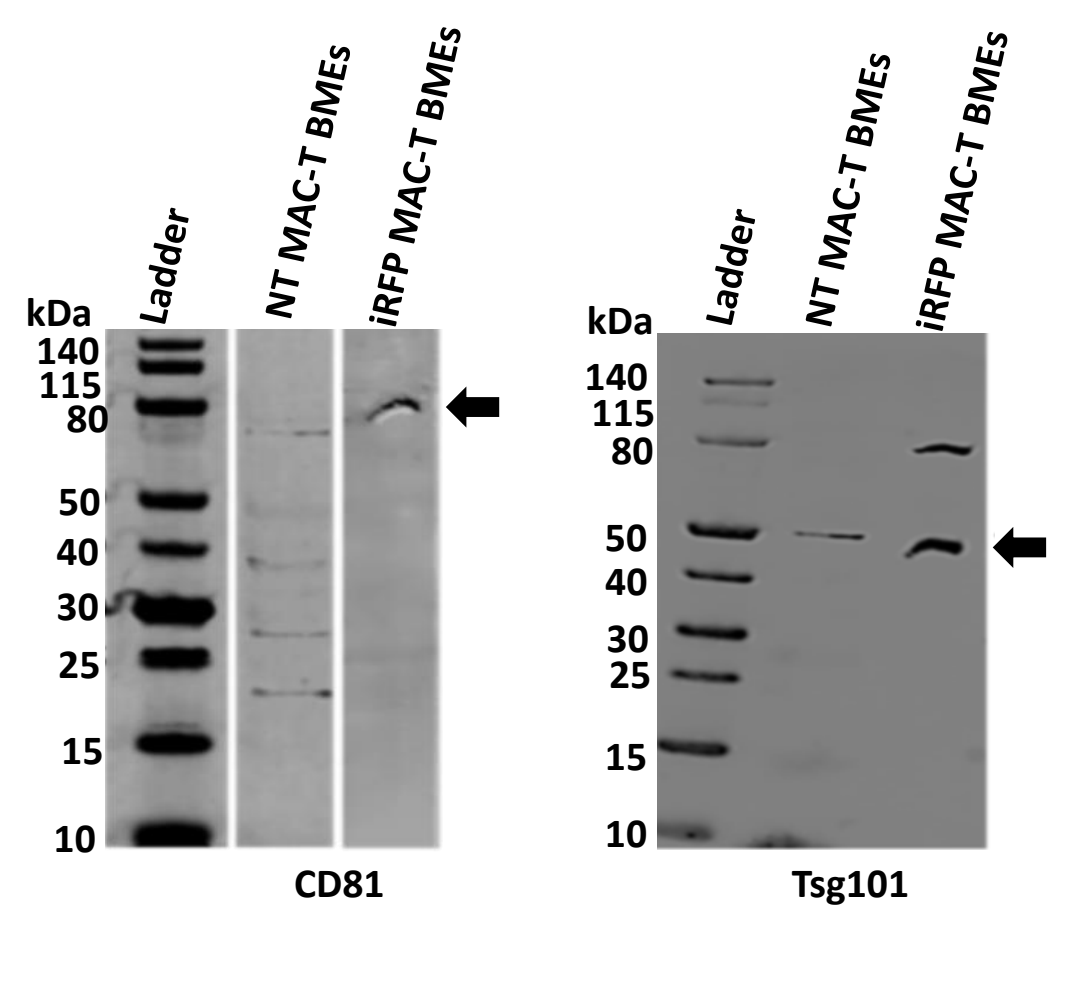

Figure 2.5 Authentication of engineered MAC-T BMEs. (A) Nanoparticle tracking analysis of non-transformed (NT) MAC-T cells and transformed MAC-T cells. Values are shown as mean \pm SEM. $\mathrm{p}>0.05(\mathrm{n}=3)$. (B) Immunoblot analysis of CD81 and Tsg101 in exosomes from nontransformed (NT) MAC-T cells and transformed MAC-T cells. 

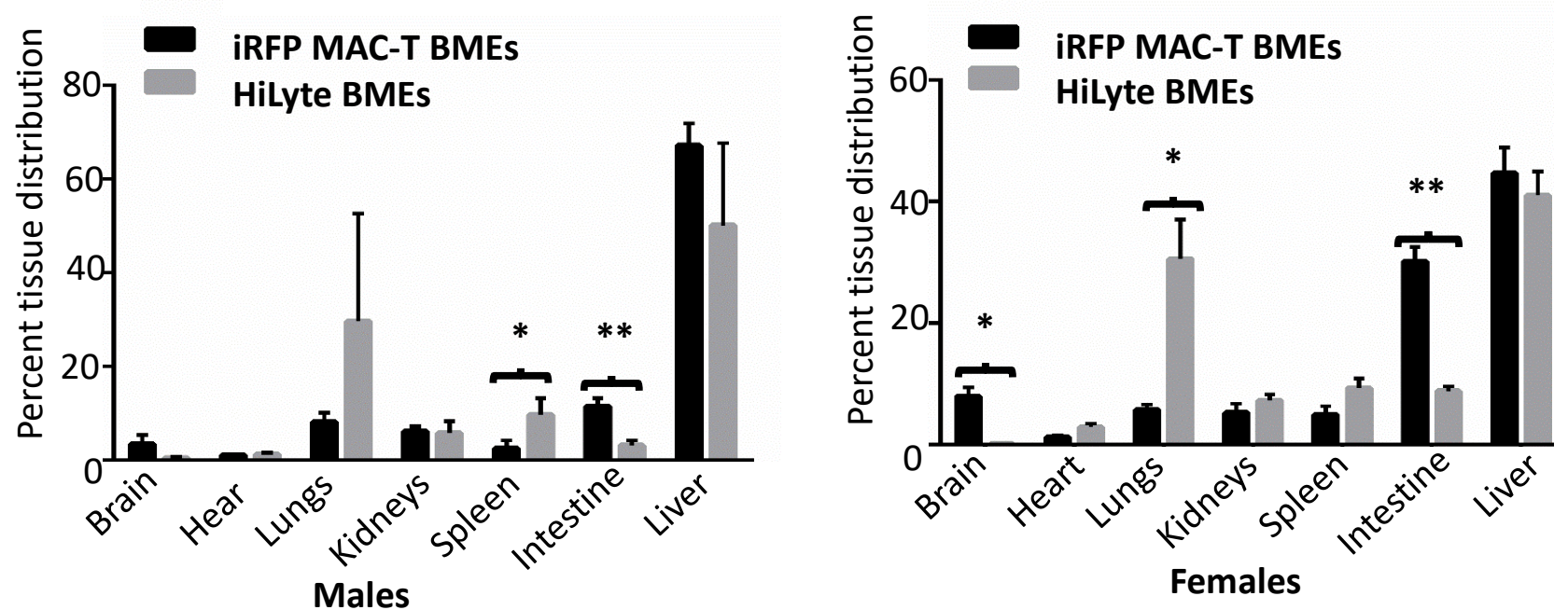

Figure 2. 6 Tissue distribution of iRFP-labeled MAC-T BMEs and HiLyte-labeled BMEs in male (left) and female (right) C57BL/6 mice. The percent tissue distribution was assessed 3 hours after intravenous injection of $1.0 \times 10^{11}$ exosomes/g body weight. Values are expressed as mean \pm SEM. $* \mathrm{p}>0.005, * * \mathrm{p}>0.0005,(\mathrm{n}=3)$. 
A

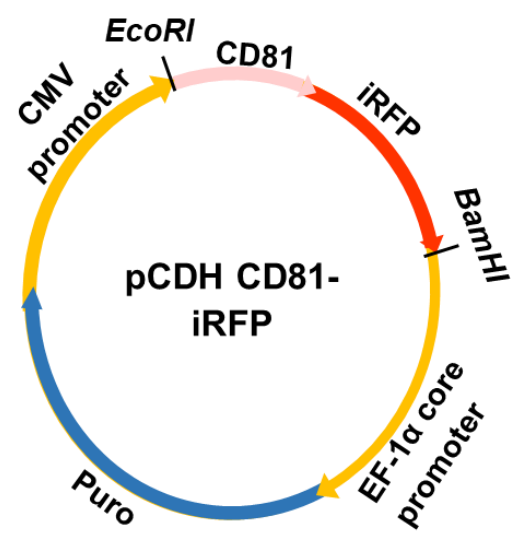

B

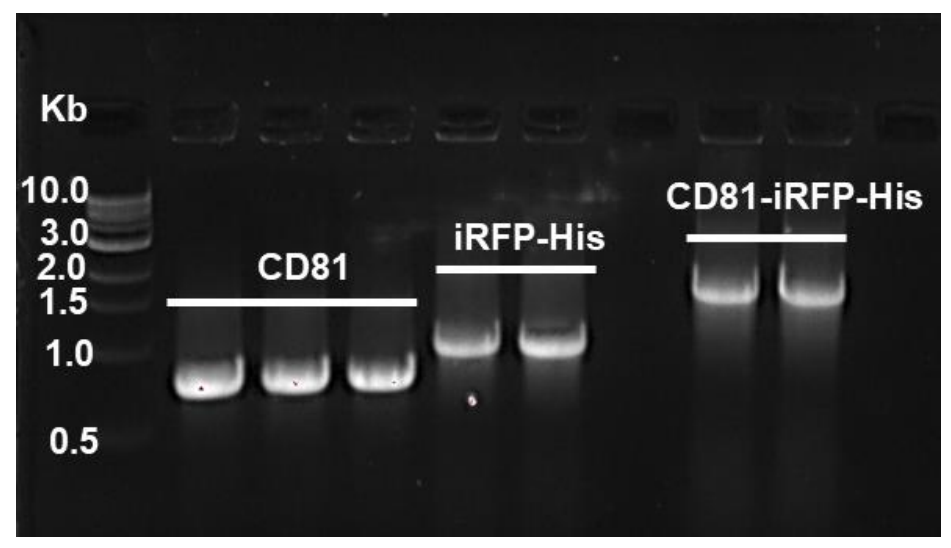

Figure S 1. Schematics illustration of CD81-iRFP vector construction. (A) Configuration of CD81-iRFP plasmid construct (B) Assembly of CD81-iRFP-His fusion protein using overlap PCR. 


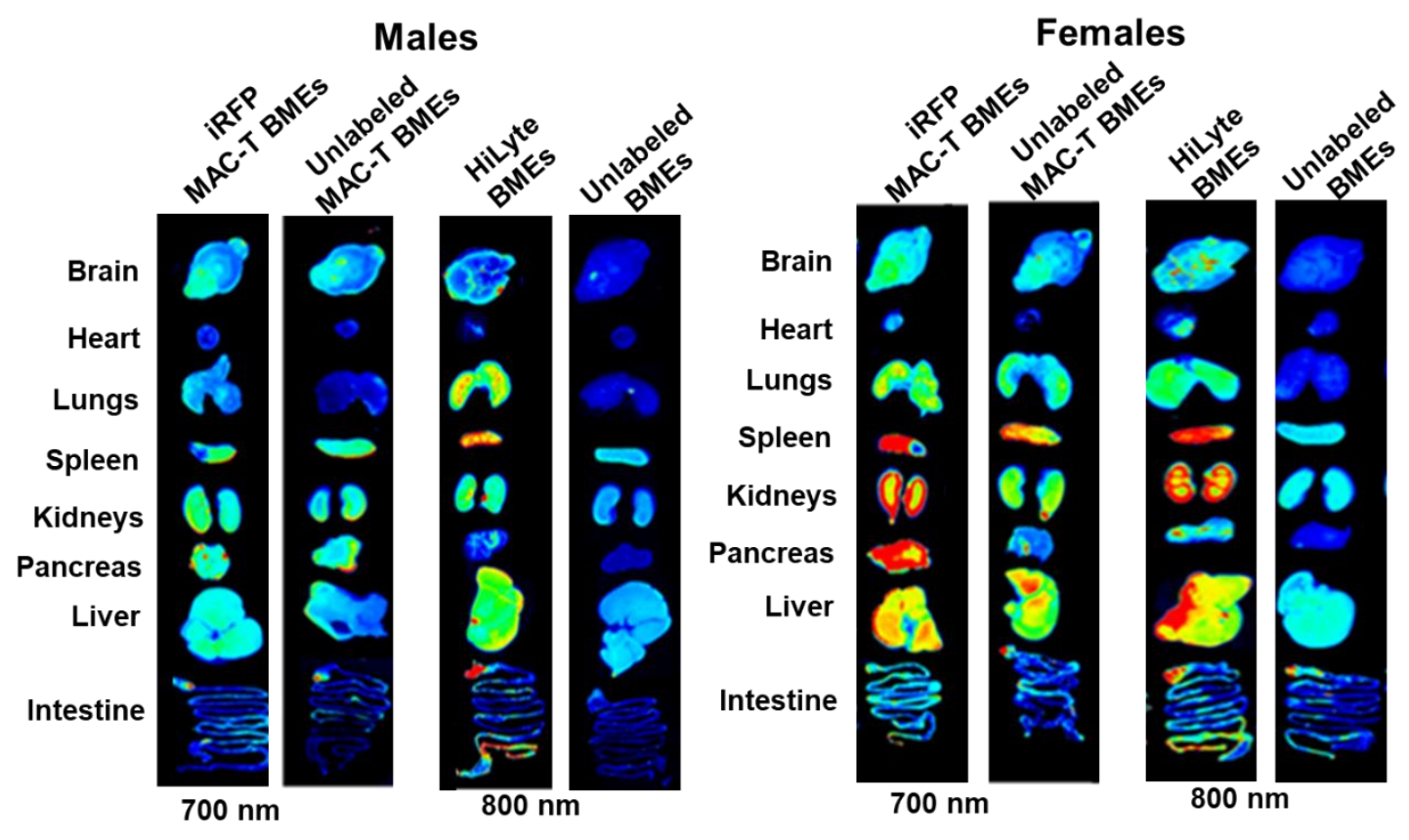

Figure S 2. Biodistribution of MAC-T BMEs and HiLyte BMEs in mice tissues. Fluorescence in excised tissues of C57BL/6 mice of after intravenous injection of iRFP MAC-T BMEs, Unlabeled MAC-T BMEs, HiLyte BMEs and unlabeled BMEs $\left(1 \times 10^{11} / \mathrm{g}\right.$ body weight $)$. 
Table 2. 1 MicroRNAs in MAC-T BMEs and BMEs.

\begin{tabular}{lllll}
\hline Samples & let-7a & miR-200c & miR-320a & miR- 1 \\
\hline MAC-T BMEs & $28.1 \pm 0.7^{\mathrm{a}}$ & $26.3 \pm 0.4^{\mathrm{a}}$ & $25.4 \pm 0.8$ & ND \\
BMEs & $23.5 \pm 1.8^{\mathrm{a}}$ & $21.4 \pm 1.2^{\mathrm{a}}$ & $24.2 \pm 1.2$ & ND \\
\hline
\end{tabular}

$*$ Value $=\mathrm{Ct}$ (cycle threshold); mean $\pm \mathrm{SEM} ; \mathrm{n}=3 ;{ }^{\mathrm{a}} p \leq 0.05$ vs BMEs.

ND, not detectable. 
Table S. 1 Primers used in real-time qPCR analysis.

\begin{tabular}{ll}
\hline MicroRNA & Forward Primer $\left(\mathbf{5}^{\prime} \mathbf{- 3}^{\prime} \mathbf{~} \mathbf{1}^{\mathbf{1}}\right.$ \\
\hline miR-320a & AAAAGCTGGGTGAGAGGGCGA \\
miR-200c & TAATACTGCCGGGTAATGATGGA \\
let-7a-5p & TGAGGTAGTAGGTTGTATAGTT \\
miR-1 & TGGAATGTAAAGAAGTATGTAT \\
miSpike & CTCAGGATGGCGGAGCGGTCT
\end{tabular}

${ }^{1}$ Qiagen's miScript Universal Primer was used as reverse primer for all reactions. miSpike is a synthetic microRNA used as internal control. 
Table S. 2 Primers used in building the CD81-iRFP-His construct.

\begin{tabular}{llc}
\hline Primer/ Template & Sequence (5'-3') & $\begin{array}{c}\text { Product } \\
\text { size (bp) }\end{array}$ \\
\hline CD81_reverse & GCCGCCCGAGCCGCCGCCTGAGGAGCCGCCGTACACCGAGCTGTT & 726 \\
iRFP-His_forward & GGCGGCTCCTCAGGCGGCGGCTCGGGCGGCGCTGAGGGAAGCGTG & 978 \\
CD81-iRFP-His_forward & GATGAATTCATGGGGGTAGAGGGCTGCACCAAG & \\
CD81-iRFP-His_reverse & GATGGATCCCTAATGATGATGATGATGATGCTCTTCCATCACGCC & 1704 \\
\hline
\end{tabular}




\section{FUTURE STUDIES}

BMEs have attracted attention as drug delivery vehicles because they are safe, scalable, and bioavailable. The drug delivery potentials of BMEs are still yet to be harnessed due to limited cargo loading and drug delivery modalities. Hence, an urgent need to explore a lab-friendly cell model that will facilitate the genetic reprogramming of BMEs for improved drug delivery. We addressed this lacuna by demonstrating that MAC-T cells secrete exosomes that are like BMEs, and these cells are amenable to genetic modifications for drug delivery studies. While our findings are a major milestone in the BMEs research community, MAC-T BMEs still merit further exploration.

Although we showed that MAC-T BMEs exhibit similar tissue distribution patterns with BMEs, we have not assessed their immunogenic effects in vivo. Induction of immune response is an important phenomenon that must be avoided in drug delivery studies as this might lead to confounding health consequences in clinical settings. Hence, this remains a fruitful line of investigation.

Additionally, our lab has shown that orally administered BMEs and their miRNA cargos are stable under harsh gastric conditions and penetrate the intestinal mucosa where they are absorbed into systemic circulation. Future studies should explore the therapeutic efficacy of MAC-T BMEs enclosed miRNA and siRNA therapeutics in the treatment of colorectal cancer and the possibilities of moving them into clinical trials considering their safety and bioavailability following oral intake.

This study sets the bar for the improvement of BMEs for targeted drug delivery through protein engineering in MAC-T cells. Given the amenability of MAC-T BMEs to genetic engineering, subsequent studies should focus on the development of trackable engineered BMEs capable of 
targeting different tissues with precision. We observed that autologous milk exosomes have higher brain accumulation compared to exogenous exosomes. Further studies need to investigate the surface protein(s) that are involved in their increased uptake and how it can be incorporated into MAC-T BMEs for improved drug delivery to the brain. Currently, our lab is actively conducting studies on genetically modified MAC-T BMEs for improved tumor targeting, increased systemic stability, and reduced intracellular degradation. Findings from this study will make therapeutics more effective in their target site. 


\section{APPENDIX}

Table 1. Nanosight analysis data for MAC-T BMEs and BMEs

\begin{tabular}{|l|l|l|l|l|l|}
\hline Samples & Replicate 1 & Replicate 2 & Replicate 3 & Mean & SEM \\
\hline MAC-T BMEs & 87.3 & 105.3 & 88.2 & 94 & 5.8 \\
\hline BMEs & 97.7 & 108.9 & 95.3 & 101 & 4.2 \\
\hline
\end{tabular}

Table 2. Nanosight analysis data for non-transformed MAC-T BMEs and transformed MAC-T BMEs.

\begin{tabular}{|l|l|l|l|l|l|}
\hline Samples & Replicate 1 & Replicate 2 & Replicate 3 & Mean & SEM \\
\hline $\begin{array}{l}\text { IRFP MAC-T } \\
\text { BMEs }\end{array}$ & 80.4 & 84.6 & 82.2 & 82 & 1.2 \\
\hline MAC-T BMEs & 87.3 & 105.3 & 88.2 & 94 & 5.8 \\
\hline
\end{tabular}

Table 3. MiRNA analysis data for MAC-T BMEs and BMEs

\begin{tabular}{|c|c|c|c|c|c|c|}
\hline & \multicolumn{3}{|c|}{ Let $7 \mathrm{a}$} & \multirow[b]{2}{*}{29.49} & \multirow[t]{2}{*}{ Mean } & \multirow[t]{2}{*}{ SEM } \\
\hline $\begin{array}{l}\text { MAC-T } \\
\text { BMEs }\end{array}$ & 29.25 & 29.70 & 29.51 & & & \\
\hline & 27.37 & 27.83 & 27.43 & 27.54 & 28.13 & 0.68 \\
\hline & 27.50 & 27.44 & 27.10 & 27.35 & & \\
\hline \multirow[t]{4}{*}{ BMEs } & 22.86 & 23.00 & 23.05 & 22.97 & & \\
\hline & 20.83 & 20.49 & 20.81 & 20.71 & 23.54 & 1.80 \\
\hline & 27.05 & 27.01 & 26.76 & 26.94 & & \\
\hline & \multicolumn{3}{|c|}{ miR200c } & & Mean & SEM \\
\hline \multirow[t]{3}{*}{$\begin{array}{l}\text { MAC-T } \\
\text { BMEs }\end{array}$} & 27.30 & 27.07 & 27.02 & 27.13 & & \\
\hline & 25.92 & 25.76 & 25.48 & 25.72 & 26.32 & 0.40 \\
\hline & 26.10 & 26.11 & 26.12 & 26.11 & & \\
\hline \multirow[t]{3}{*}{ BMEs } & 21.17 & 21.52 & 21.31 & 21.33 & & \\
\hline & 19.20 & 19.19 & 19.24 & 19.21 & 21.35 & 1.23 \\
\hline & 23.52 & 23.37 & 23.60 & 23.50 & & \\
\hline \multirow[t]{4}{*}{$\begin{array}{l}\text { MAC-T } \\
\text { BMEs }\end{array}$} & \multicolumn{3}{|c|}{ miR320a } & & Mean & SEM \\
\hline & 24.71 & 24.42 & 23.97 & 24.37 & & \\
\hline & 24.88 & 24.60 & 25.06 & 24.85 & 25.39 & 0.79 \\
\hline & 26.96 & 27.05 & 26.87 & 26.96 & & \\
\hline \multirow[t]{3}{*}{ BMEs } & 23.94 & 24.04 & 24.02 & 24.00 & & \\
\hline & 22.23 & 22.29 & 22.36 & 22.29 & 24.22 & 1.18 \\
\hline & 26.29 & 26.49 & 26.35 & 26.38 & & \\
\hline
\end{tabular}


Table 4. Percent tissue distribution data in male and female mice.

\begin{tabular}{|c|c|c|c|c|c|c|c|c|c|c|}
\hline \multicolumn{11}{|c|}{ Female Percent Tissue distribution } \\
\hline & \multicolumn{5}{|c|}{ iRFP MAC-T BMEs } & \multicolumn{5}{|c|}{ HiLyte BMEs } \\
\hline & Replicates & & & Mean & SEM & \multicolumn{3}{|c|}{ Replicates } & Mean & SEM \\
\hline Brain & 5.04 & 9.24 & 9.61 & 7.96 & 1.47 & 0.09 & 0.12 & 0.12 & 0.11 & 0.01 \\
\hline Heart & 0.73 & 1.13 & 1.76 & 1.21 & 0.30 & 2.88 & 3.88 & 1.90 & 2.89 & 0.57 \\
\hline Lungs & 7.16 & 4.39 & 5.82 & 5.79 & 0.80 & 36.37 & 17.61 & 37.68 & 30.55 & 6.48 \\
\hline Kidneys & 3.39 & 8.16 & 4.31 & 5.29 & 1.46 & 5.20 & 8.19 & 8.38 & 7.26 & 1.03 \\
\hline Spleen & 2.09 & 6.41 & 6.26 & 4.92 & 1.42 & 6.68 & 12.04 & 9.27 & 9.33 & 1.55 \\
\hline $\begin{array}{l}\text { Intestinal } \\
\text { tract }\end{array}$ & 30.70 & 33.95 & 25.78 & 30.14 & 2.37 & 9.40 & 9.71 & 7.33 & 8.81 & 0.75 \\
\hline Liver & 50.90 & 36.71 & 46.45 & 44.69 & 4.19 & 39.37 & 48.45 & 35.32 & 41.05 & 3.88 \\
\hline \multicolumn{11}{|c|}{ Male Percent Tissue Distribution } \\
\hline & \multicolumn{5}{|c|}{ iRFP MAC-T BMEs } & \multicolumn{5}{|c|}{ HiLyte BMEs } \\
\hline & \multicolumn{3}{|c|}{ Replicates } & Mean & SEM & \multicolumn{3}{|c|}{ Replicates } & Mean & SEM \\
\hline Brain & 2.96 & 1.71 & 5.58 & 3.42 & 1.14 & 0.35 & 0.33 & 0.70 & 0.46 & 0.12 \\
\hline Heart & 0.95 & 1.01 & 1.35 & 1.10 & 0.12 & 1.63 & 1.03 & 1.03 & 1.23 & 0.20 \\
\hline Lungs & 7.13 & 6.91 & 10.42 & 8.15 & 1.14 & 38.28 & 47.04 & 3.57 & 29.63 & 13.27 \\
\hline Kidneys & 4.98 & 7.10 & 6.32 & 6.13 & 0.62 & 4.07 & 4.47 & 8.68 & 5.74 & 1.47 \\
\hline Spleen & 0.86 & 4.29 & 2.28 & 2.48 & 1.00 & 7.34 & 7.86 & 13.80 & 9.67 & 2.07 \\
\hline $\begin{array}{l}\text { Intestinal } \\
\text { tract }\end{array}$ & 10.57 & 13.50 & 10.53 & 11.53 & 0.98 & 2.37 & 4.33 & 2.85 & 3.18 & 0.59 \\
\hline Liver & 72.54 & 65.48 & 63.51 & 67.18 & 2.74 & 45.95 & 34.94 & 69.38 & 50.09 & 10.16 \\
\hline
\end{tabular}

